\title{
Coarse Competitive Equilibrium and Extreme Prices*
}

\author{
Faruk Gul ${ }^{\dagger}$ \\ Wolfgang Pesendorfer \\ Tomasz Strzalecki ${ }^{\S}$
}

\begin{abstract}
We introduce a notion of coarse competitive equilibrium, to study agents' inability to tailor their consumption to prices. Our goal is to incorporate limited cognitive ability (in particular limited attention, memory and complexity) into the analysis of competitive equilibrium. Compared to standard competitive equilibrium, our concept yields more extreme prices and, when all agents have the same endowment, riskier allocations. We provide a tractable model suitable for general equilibrium analysis as well as asset pricing.
\end{abstract}

\footnotetext{
*Date: September 2, 2016. This research was supported by grants from the Sloan Foundation and the National Science Foundation SES-0010394 and SES-1123729 and the NSF CAREER grant SES-1255062. We thank a coeditor and three anonymous referees for very helpful comments, as well as Nicolas Butler and Kevin He for expert proofreading. The authors declare that they have no relevant or material financial interests that relate to the research described in this paper.

†Princeton University. Email: fgul@princeton.edu

${ }^{\ddagger}$ Princeton University. Email: pesendor@princeton.edu

${ }_{\S}^{\S}$ Harvard University. Email: tomasz_strzalecki@harvard.edu
} 


\section{Introduction}

In standard consumer theory, agents adjust their consumption whenever prices change. In this paper, we consider agents restricted to coarse consumption plans. Such agents partition the possible prices into finitely many categories and adjust their consumption only when the price moves from one category to another. Consumers form their price categories optimally - that is, choose them to maximize their ex ante utility.

Consider, for example, an agent who forms two categories. This agent partitions prices into a set of high prices and a set of low prices, then chooses one consumption level for each cell of the partition. Thus, this agent makes two decisions: how to define the binary partition and how much to consume in each partition cell. The second decision is a standard optimization problem with incomplete information. The first is our device for modeling how attention allocation responds to economic incentives.

To simplify the exposition, we assume that the economy has two periods, a planning period and a consumption period, and that there is a single physical good. ${ }^{1}$ Households learn the price after the planning period and before the consumption period. In the planning period, each agent chooses an optimal partition of prices and an optimal consumption level for each partition cell. We assume that agents are expected utility maximizers with a CRRA utility index and show that every cell of an optimal partition is an interval of consecutive prices.

Optimally partitioning the state space may be difficult; however, our model is not meant as a description of an agent's reasoning process. Rather, we interpret the optimal partition as the outcome of an adjustment process in which the agent gradually adjusts cognitive resources to increase her utility. Our goal is to capture an agent who is unable to react to all price changes, but responds to incentives when allocating her limited cognitive resources.

We analyze an endowment economy with a continuum of agents, each subject to the coarseness constraint described above. We refer to the resulting equilibrium as a coarse competitive equilibrium. We show that it exists and is Pareto efficient (given the restriction to coarse consumption plans). In a coarse competitive equilibrium, ex ante identical agents must choose distinct plans and, as a result, consumption tends to be more risky than in a standard compet-

\footnotetext{
${ }^{1}$ We extend the model to an infinite horizon in the online appendix. We briefly discuss this extension in section 5.5.
} 
itive equilibrium. $^{2}$

We fix the agents' utility functions and consider a sequence of discrete endowment economies. We show that coarse competitive equilibrium prices become extreme as the economy approaches a continuous limit. The price of consumption goes to infinity when the endowment is at or near the lower bound of the distribution; it converges to zero when the endowment is at or near the upper bound.

To see the intuition behind our extreme-price result, consider an economy with coarseness constraint $k=2$. As in the standard case, equilibrium prices in a coarse competitive equilibrium decrease as aggregate endowment increases. Moreover, each agent optimally partitions prices into two intervals, a low (price) interval and a high (price) interval. Since aggregate endowment varies and each agent consumes the same amount at all prices in a given interval, market clearing requires some agents to designate a small interval in the upper tail of the price distribution as their high interval. For those agents, a price in the high interval is unlikely whereas a price in the low interval occurs with high probability. Other agents must do the opposite and specify a small interval in the lower tail of the price distribution as their low interval. For those agents, a price in the low interval is unlikely. Agents in either of these groups allocate a valuable resource, a partition cell, to an unlikely event. To render this decision optimal, prices must ensure that these agents are adequately compensated. Households who pay attention to the highest prices benefit if these prices are significantly higher than the average of the other prices. They do so by consuming little, selling most of their endowment and increasing their consumption in the low interval of their partitions. Conversely, households who pay attention to the lowest prices benefit if those prices are low enough to enable them to consume large amounts at those (and only those) low prices. Thus, in a coarse competitive equilibrium prices must be lower (higher) than in a standard competitive equilibrium when the aggregate endowment is high (low).

Our analysis highlights a particular mechanism behind extreme prices: market clearing requires that some consumers pay attention to prices even in very unlikely states. For consumers to have the incentive to allocate their scarce cognitive resources to such unlikely events, prices must be sufficiently volatile. This mechanism is robust to various modeling assumptions. In the last section of the paper, we examine how our results would change if agents were differentiated

\footnotetext{
${ }^{2}$ This is true, for example, whenever all agents have the same endowment.
} 
by their risk posture and their complexity constraint; if instead of coarse consumption we assumed a coarseness constraint on net trades; and, the extent to which our conclusions depend on constant relative risk aversion.

\subsection{Relation to Literature}

The game theory literature offers strategic analogs of coarse equilibrium. Neyman (1985), Rubinstein (1986), and Abreu and Rubinstein (1988) limit players' strategies in a repeated game to those implementable by finite state automata. Our approach is closest to Neyman (1985) who studies Nash equilibria of a game in which the number of states in the automaton is bounded. Rather than restricting the set of repeated game strategies, Abreu and Rubinstein (1988) assume that more complex strategies are more costly. Rubinstein (1986) examines a lexicographic cost of complexity and imposes a version of subgame perfection which precludes agents from adopting a different automaton later in the game. Jehiel (2005) and Jehiel and Samet (2007) require players to respond identically in similar situations by bundling their decision nodes into exogenous analogy classes. Mengel (2012) studies the evolutionary dynamics of categorization. He assumes a fixed marginal cost per partition cell and that optimal partition size is determined in equilibrium.

Decision makers with a coarse understanding of the state space also appear in the choice theory literature, for example, in Ahn and Ergin (2010), Dekel et al. (2001), and Epstein et al. (2007). In Masatlioglu et al. (2012), agents make optimal choices subject to an endogenous attention constraint. In Dow (1991)'s model of search with limited memory, the agent optimally partitions histories. Piccione and Rubinstein (1997) examine the relationship between limited memory (i.e., imperfect recall) and time consistency. Fryer and Jackson (2008) show how optimal categorization can lead to statistical discrimination against minorities. Similarly, Wilson (2014) analyzes long-run inference and shows that the optimal use of a limited memory can lead to many well-studied behavioral biases. Mohlin (2014) studies optimal categorization in prediction tasks; in his model, a bias-variance tradeoff determines the cost and benefit of a partition. Mullainathan (2002) studies a model of coarse categorization and its implications for asset returns and trade volume.

Coarse behavior is also at the heart of the recent literature on rational inattention, which 
focuses on how information processing frictions impact asset prices and responses to monetary policy. Sims (2003) assumes that agents allocate their attention optimally subject to an information-theoretic constraint. We focus on partitional information structures and limit the number of possible signal values instead of using the entropy based constraint. Woodford (2012) modifies Sims' cost function to address consumer choice anomalies; the restriction of his model to partitional information leads to a constraint similar to ours. Ellis (2015) studies general cost functions for partitional information structures; his constrained attention model provides an axiomatic foundation for our work. Mankiw and Reis (2002) study a model in which only a fraction of agents obtain new information each period. Gabaix (2014) solves a quadratic approximation to the optimal attention problem, adapted from the lasso method in statistics (Tibshirani, 1996), and shows that it may lead to volatile prices.

Despite the differences in modeling details, all these papers, including ours, constrain agents' ability to tailor their behavior to their environment. Our coarseness constraint implements this idea in a way that yields a tractable competitive equilibrium model.

\section{Coarse Consumers}

Let $N=\{1, \ldots, n\}$ be the states of the world. There is one physical good; a consumption plan $c$ is a vector in $\mathcal{C}=\mathbb{R}_{+}^{n}$ that determines how much of the good the agent consumes in each state. A price $p$ is an element of the $n-1$-dimensional simplex $\Delta(N)=\left\{p \in \mathbb{R}_{+}^{n}: \sum_{i} p_{i}=1\right\}$. For a given budget $w>0$, the budget-feasible consumption plans are $B(p, w)=\{c \in \mathcal{C}: p \cdot c \leq w\}$.

Feasible consumption plans are those that satisfy the budget constraint above as well as the following attention constraint. Let $\mathcal{P}(k)$ be the collection of partitions of $N$ into $k$ cells. A consumption plan is coarse if it is measurable with respect to some partition $S=\left\{S_{1}, \ldots, S_{k}\right\} \in \mathcal{P}(k){ }^{3}$ The partition $S$ specifies how the consumer allocates her attention and once she chooses attentions strategy $S$, the consumer is limited to consumption plans that are $S$-measurable; that is, plans $c$ such that $c_{i}=c_{j}$ for all states $i$ and $j$ that are in the same cell of $S$. Once her attention strategy is determined, the consumer maximizes utility

\footnotetext{
${ }^{3}$ We assume that $k$ is a fixed. Alternatively, one could let the agent choose $k$ at a cost. A similar modeling decision arises in the rational inattention literature, c.f., Sims (2003) and Woodford (2012). We discuss the costly $k$ version of our model in more detail in section 5.2.
} 
among all feasible consumption plans consistent with this attention strategy; that is, she solves the following maximization problem:

$$
V_{S}(p, w)=\max _{c \in \mathcal{C}_{S} \cap B(p, w)} \sum_{i=1}^{n} u\left(c_{i}\right) \pi_{i}
$$

where $\pi_{i}$ is the prior probability of state $i$ and $u$ is a strictly concave CRRA utility index with coefficient of relative risk aversion $\rho>0$ :

$$
u\left(c_{i}\right)= \begin{cases}c_{i}^{1-\rho} /(1-\rho) & \text { if } \rho \neq 1 \\ \ln c_{i} & \text { if } \rho=1\end{cases}
$$

We also assume that the consumer chooses her attention strategy optimally; that is, $S$ solves the following maximization problem:

$$
V(p, w)=\max _{S \in \mathcal{P}(k)} V_{S}(p, w)
$$

Thus, consumers in our model make two decisions: first they decide how to allocate their attention (choose $S \in \mathcal{P}(k)$ ); then, they choose an optimal consumption consistent with their attention strategy and their budget (choose $c \in \mathcal{C}_{S} \cap B(p, w)$ ). As in a standard economy without the attention constraint, each consumer trades $N$ distinct goods. The choice of $S$ implies that the consumer demands the same quantity of consumption for any pair of states in the same cell of $S$; thus, her demand is $S$-measurable. In other words, the consumer behaves as if goods that belong to the same cell of of her partition are perfect complements.

It is convenient to express the maximization problem above as a one-step problem: any coarse consumption plan has at most $k$ distinct consumption levels and, conversely, for any consumption plan with $k$ or fewer distinct consumption levels there is some partition $S \in \mathcal{P}(k)$ that renders it $S$-measurable. Formally,

Definition 1. The consumption plan $c \in \mathcal{C}$ is coarse if the collection $\left\{c_{i}\right\}_{i \in N}$ has at most $k$ distinct elements.

We write $\mathcal{C}_{k}$ for the set of all $k$-coarse consumption plans. Then, the budget set of a 
consumer who is subject to the attention constraint is

$$
B_{k}(p, w)=\left\{c \in \mathcal{C}_{k}: p \cdot c \leq w\right\}
$$

Let $U(c):=\sum_{i=1}^{n} u\left(c_{i}\right) \pi_{i}$. The consumer's problem can be restated as follows:

$$
V(p, w)=\max _{c \in B_{k}(p, w)} U(c)
$$

Let $D_{k}(p, w)$ denote the set of optimal plans; that is, solutions to (2) for a fixed CRRA utility index $u$.

\subsection{Optimal Plans}

In Theorem 1 below, we show that when studying optimal consumption plans, we may restrict attention to partitions that correspond to price ranges.

Definition 2. A consumption plan $c$ is monotone if $\frac{p_{i}}{\pi_{i}}>\frac{p_{j}}{\pi_{j}}$ implies $c_{i} \leq c_{j}$. The plan $c$ is measurable if $\frac{p_{i}}{\pi_{i}}=\frac{p_{j}}{\pi_{j}}$ implies $c_{i}=c_{j}$.

The following theorem shows that the agent always chooses a consumption plan that is monotone and measurable, i.e., she consumes more in states that have lower (normalized) prices and the same amount in states that have the same price.

Theorem 1. Any $c \in D_{k}(p, w)$ is monotone and measurable.

To gain intuition for Theorem 1, consider a consumer with wealth 1. Given a partition $S=\left(S_{1}, \ldots, S_{k}\right)$, let $p(l)$ and $\pi(l)$ be the total price and probability of cell $S_{l}$. That is, $p(l)=\sum_{j \in S_{l}} p_{j}$ and $\pi(l)=\sum_{j \in S_{l}} \pi_{j}$. Using standard indirect utility calculations, we can write (a monotone transformation of) the consumer's maximal utility when choosing $S$ as:

$$
V_{\sigma}(S)=\sum_{j=1}^{k} p(j) \psi_{\sigma}\left(\frac{\pi(j)}{p(j)}\right)
$$

where $\sigma=1 / \rho$ is the coefficient of relative risk aversion. The key to the proof is that $\psi_{\sigma}$ is a strictly convex function. To see how convexity of $\psi_{\sigma}$ implies the result, let $k=2, n=3$ and 
assume $\frac{p_{1}}{\pi_{1}}>\frac{p_{2}}{\pi_{2}}>\frac{p_{3}}{\pi_{3}}$. For the partition $S$ that lumps together states 1 and $3, V_{\sigma}(S)=E_{q}\left[\psi_{\sigma}\right]$, where $E_{q}\left[\psi_{\sigma}\right]$ is the expectation of $\psi_{\sigma}$ given the probability distribution $q$ which yields $\frac{\pi_{1}+\pi_{3}}{p_{1}+p_{3}}$ with probability $p_{1}+p_{3}$ and $\frac{\pi_{2}}{p_{2}}$ with probability $p_{2} \cdot{ }^{4}$ Similarly, for the partition $S^{\prime}$ that lumps together states 1 and $2, V_{\sigma}\left(S^{\prime}\right)=E_{q^{\prime}}\left[\psi_{\sigma}\right]$ where $q^{\prime}$ yields $\frac{\pi_{1}+\pi_{2}}{p_{1}+p_{2}}$ with probability $p_{1}+p_{2}$ and $\frac{\pi_{3}}{p_{3}}$ with probability $p_{3}$; for the partition $S^{\prime \prime}$ that lumps together states 2 and $3, V_{\sigma}\left(S^{\prime \prime}\right)=E_{q^{\prime \prime}}\left[\psi_{\sigma}\right]$ where $q^{\prime \prime}$ yields $\frac{\pi_{2}+\pi_{3}}{p_{2}+p_{3}}$ with probability $p_{2}+p_{3}$ and $\frac{\pi_{1}}{p_{1}}$ with probability $p_{1}$. It is easy to see that $q^{\prime}$ or $q^{\prime \prime}$ is a mean-preserving spread of $q$. Then, the strict convexity of $\psi_{\sigma}$ implies that either $S^{\prime}$ or $S^{\prime \prime}$ will yield a higher utility than $S$. A slightly modified version of the preceding argument shows that if $p_{1}=p_{2}>p_{3}$, then the only optimal partition is $S^{\prime}=(\{1,2\},\{3\})$.

\subsection{Interpreting the Coarseness Constraint}

In section 3, we analyze the competitive equilibria of an endowment economy in which consumers choose coarse consumption plans. In this subsection, we discuss how the coarseness restriction may be interpreted as a cognitive constraint and how an economy with coarse consumers might function.

In any economy, each consumer solves two separate problems: first, she determines which plans she can afford at the prevailing prices given her endowment; then, she chooses among the affordable plans. We assume consumers rationally solve the first problem but are boundedly rational when solving the second.

This particular type of bounded rationality can be justified as follows. Consider a consumer whose transactions are credited or debited to a single bank account. At the end of the month, the consumer's paycheck (i.e., the value of her endowment) is credited; throughout the month, her purchases and withdrawals are debited. We assume that the consumer cannot tailor her everyday consumption exactly to price fluctuations; instead, she has a default bundle that she purchases every period unless the price of that bundle falls outside the normal range. She switches to a high-price bundle if the normal bundle's price exceeds an upper threshold and she switches to the low-price bundle if its price falls below a lower threshold. Such a consumer responds to a range of prices with the same consumption decision and ends up with a 3-coarse consumption plan. The consumer's price thresholds will depend on her risk attitude, her budget

\footnotetext{
${ }^{4}$ Recall that we have normalized prices to sum up to 1.
} 
and on the distribution of equilibrium prices. In a stationary environment, the consumer would gradually learn which combinations of thresholds and bundles meet her needs and satisfy her budget constraint. Moreover, changes in the distribution of prices would lead to changes in the choice of thresholds. That is, the way the consumer partitions prices into constant consumption categories would be sensitive to price incentives. Our idealized formulation captures this price sensitivity by having consumers choose their thresholds and consumption bundles optimally.

In an economy with coarse consumers, the price mechanism functions just like in a standard economy. To see the similarity, consider an example with three equally likely endowment states, $k=2$, and logarithmic utility index, so that the consumers have the following utility function (up to normalization):

$$
u\left(c_{1}, c_{2}, c_{3}\right)=\ln c_{1}+\ln c_{2}+\ln c_{3} .
$$

The aggregate endowment in state $i$ is strictly lower than in state $i+1$. In this setting, only two partitions can be optimal: $\{\{1,2\},\{3\}\}$ and $\{\{1\},\{2,3\}\}$. Suppose that mass $m$ of consumers choose the first of these partitions and mass $1-m$ chooses the second one (call them group 1 and group 2). Hence, everyone in group 1 (group 2) will consume the same quantity in states 1 and 2 (2 and 3). Optimal consumption and utility at any price vector for consumers in groups 1 and 2, respectively, will be identical to the optimal consumption and utility of a standard consumer with utility functions $v_{1}, v_{2}$, where

$$
\begin{gathered}
v_{1}\left(c_{1}, c_{2}, c_{3}\right)=2 \min \left\{\ln c_{1}, \ln c_{2}\right\}+\ln c_{3} \\
v_{2}\left(c_{1}, c_{2}, c_{3}\right)=\ln c_{1}+2 \min \left\{\ln c_{2}, \ln c_{3}\right\} .
\end{gathered}
$$

Therefore, choosing a partition amounts to choosing a utility function with perfect complements. Once the household partitions are specified, every consumer maximizes utility subject to a standard budget constraint. Putative equilibrium prices are chosen to clear markets. For these putative equilibrium prices to be coarse equilibrium prices, no household may have an incentive to change its partition. Otherwise, some members from one group would change their partition causing $m$ to change; in that case, market clearing prices will be chosen again and households will assess their partitions. ${ }^{5}$ This process will continue until every household is satisfied with

\footnotetext{
${ }^{5}$ We envision a continuous adjustment process where a small fraction of households has the opportunity to
} 
both its partition and consumption choices and markets clear.

One alternative to our coarse consumption model is the coarse net trades model. In the latter, the quantity bought or sold at each price would have to satisfy the coarseness constraint. Which model is more appropriate depends on the application. When analyzing household choices, it is natural to assume that cognitive limitations translate into infrequent changes in consumption; that is, coarse consumption. When analyzing portfolio choice problems, it is natural to assume that cognitive limitations lead to infrequent trading; i.e., coarse net trades. With coarse net trades, the equilibrium is sensitive to the distribution of the aggregate endowment. For example, if the initial endowment is Pareto efficient, then the equilibrium of the coarse net trades will be a standard competitive equilibrium. Nevertheless, we conjecture that if household's endowments are "sufficiently far" from any possible equilibrium consumption plan, then the conclusions of our main theorem continue to hold. In section 5.3 we identify a simple condition on the endowment distribution that ensures extreme prices in an economy with coarse net trades.

\section{Coarse Competitive Equilibria}

In this section, we analyze an endowment economy consisting of a continuum of consumers who have a common CRRA utility function $u$ and a common prior $\pi$ on $N$ but have idiosyncratic endowments. Since the preferences are homothetic, the set of optimal consumption plans are homogeneous of degree one in wealth:

$$
D_{k}(p, w)=\left\{w \cdot c \mid c \in D_{k}(p, 1)\right\}
$$

where $w=p \cdot \omega$ is the value of this consumers endowment $\omega$ at prices $p$ and $D_{k}(p, 1)$ is the optimal consumption plans of a consumer with wealth 1 facing prices $p$. However, unlike in the corresponding standard economy without the coarseness constraint, budget sets $B_{k}(p, w)$ are not convex and therefore the set of optimal consumption plans $D_{k}(p, w)$ is not a singleton. Nevertheless, despite the coarseness constraint, homotheticity ensures that the distribution of

adjusts their partitions at every time $t$. We have not analyzed the convergence properties of this stochastic process. 
endowments does not affect the set of competitive equilibrium prices: this set depends only on the aggregate endowment. Therefore, since our focus is on equilibrium prices, we will suppress individual endowments throughout the subsequent discussion. ${ }^{6}$

Let $s=\left(s_{1}, \ldots, s_{n}\right)$ be the aggregate endowment. We write $a$ for the smallest value of $s_{i}, b$ for the largest, and assume that $0<a<b$. If the aggregate endowment has $k$ or fewer distinct values, then the consumer's complexity constraint does not bind. In that case, the economy has a standard competitive equilibrium in which every agent's consumption is a multiple of the aggregate endowment. On the other hand, if $k=1$; that is, if all consumers must choose the same consumption in every state, then in a competitive equilibrium aggregate consumption will equal the minimum endowment $a$, in every state. To avoid these trivial cases, we assume that $k$ is greater than 1 but smaller than the number of distinct values of $s$.

The coarseness constraint ensures that even in a uniform economy, consumptions would differ in equilibrium. Otherwise, aggregate consumption would take on at most $k$ distinct values. Since the aggregate endowment has more than $k$ distinct values this would mean that in some states endowment would be wasted. As we show below, this is typically not optimal.

Let $\Delta$ denote the set of functions $\mu: \mathcal{C}_{k} \rightarrow[0,1]$ such that, $K(\mu)=\{c: \mu(c)>0\}$, the support of $\mu$, is finite and $\sum_{c \in \mathcal{C}_{k}} \mu(c)=1$. Elements of $\Delta$ are allocations and $\mu(c)$ is the mass of agents who choose plan $c \in \mathcal{C}_{k}$. The allocation $\mu$ is feasible if the average consumption is less than or equal to the per capita endowment in every state; that is, if for all $i \in N$,

$$
\Sigma_{i}(\mu):=\sum_{c \in K(\mu)} c_{i} \cdot \mu(c) \leq s_{i}
$$

Definition 3. A coarse competitive equilibrium is a price $p$ and a feasible allocation $\mu$ such that all plans in the support of $\mu$ solve the maximization problem (2) at prices $p$ and wealth $s \cdot p$; that is, $\mu(c)>0$ implies $c \in D_{k}(p, s \cdot p)$.

We say that two consumption plans $c, c^{\prime}$ conform if they induce the same partition of the state space; that is, $c_{i}=c_{j}$ if and only if $c_{i}^{\prime}=c_{j}^{\prime}$. The following four properties of equilibrium

\footnotetext{
${ }^{6}$ Endowments only play a role in the definition of a competitive equilibrium allocation below and hence in Theorem 2(ii). To facilitate the simple characterization in Theorem 2(ii), we state the definition of an equilibrium for an economy in which all agents have the same endowment. The corresponding equilibrium price will also be an equilibrium price for any endowment distribution provided every agent's wealth at this price is strictly greater than 0 .
} 
play a key role in our analysis:

Definition 4. An allocation $\mu$ is

(i) simple if $c, c^{\prime} \in K(\mu), c \neq c^{\prime}$ implies $c$ and $c^{\prime}$ do not conform.

(ii) fair if $c, c^{\prime} \in K(\mu)$ implies $U(c)=U\left(c^{\prime}\right)$.

(iii) monotone if for all $c \in K(\mu), c_{i} \geq c_{j}$ whenever $s_{i}>s_{j}$.

(iv) measurable if for all $c \in K(\mu), c_{i}=c_{j}$ whenever $s_{i}=s_{j}$.

In a simple allocation, every equilibrium attention strategy has at most one consumption plan associated with it. Thus, if $\mu$ is simple, the cardinality of $K(\mu)$ is at most equal to the number of partitions in $\mathcal{P}(k)$. In a fair allocation, every agent has the same utility. In a monotone allocation, every agent's consumption increases weakly in the aggregate endowment, and in a measurable allocation, every agent's consumption is a function of the aggregate endowment.

The mean utility, $W(\mu)$, of allocation $\mu$ is

$$
W(\mu)=\sum_{c} U(c) \cdot \mu(c)
$$

We say that a feasible allocation $\mu$ solves the planner's problem if $W(\mu) \geq W\left(\mu^{\prime}\right)$ for all feasible allocations $\mu^{\prime}$. The main result of this section is Theorem 2 below, which identifies properties of the solutions to the planner's problem and relates it to coarse competitive equilibria of the economy in which all agents have identical endowments. In an economy without our coarseness constraint, simplicity, fairness, monotonicity and measurability of solutions to the planner's problem follows immediately from the strict concavity of the utility function. The argument for simplicity is unaffected by coarseness constraint: the average of two plans that are measurable with respect to the same partition is feasible and yields a higher utility than at least one of the original plans. However, none of the remaining properties hold for a general strictly concave utility function under the coarseness constraint. Theorem 2 shows that they do hold with a strictly concave CRRA utility function. 
Theorem 2. (i) There is a solution to the planner's problem and every solution to the planner's problem is simple, fair, monotone, and measurable. (ii) An allocation solves the planner's problem if and only if it is a coarse competitive equilibrium allocation.

Existence and Pareto efficiency of a coarse competitive equilibrium requires neither CRRA preferences nor the correspondence between solutions to the planner's problem and equilibria. As long as utility functions are continuous, existence can be established using a fixed-point argument. However, such a proof would not yield the monotonicity and measurability of equilibrium allocations. As we show in section 5, without CRRA utility, it is possible to construct examples of coarse competitive equilibria that do not satisfy these properties. The first welfare theorem holds provided preferences are locally nonsatiated. Of course, Pareto efficiency must be defined relative to feasible coarse allocations.

The final result of this section shows that equilibrium prices are essentially unique and monotone. A price $p$ is monotone if greater aggregate endowment implies a weakly lower pricing kernel; that is, $\frac{p_{i}}{\pi_{i}} \leq \frac{p_{j}}{\pi_{j}}$ whenever $s_{i}>s_{j}$. In a pure endowment economy the realized endowment resolves all uncertainty; that is, $s_{i} \neq s_{j}$ whenever $i \neq j$. In that case, the equilibrium price is unique and monotone. If there is more than one state with a given endowment, the equilibrium price need not be unique; however, the sum of the prices for a given endowment is unique. For any $r \in\left\{s_{i}: i \in N\right\}$, let $p(r)=\sum_{\left\{i: s_{i}=r\right\}} p_{i}$. Two prices $p, \hat{p}$ are equivalent if $p(r)=\hat{p}(r)$ for all $r$. We say that the price is essentially unique if all equilibrium prices are equivalent.

Theorem 3. The coarse competitive equilibrium price of any economy is essentially unique and monotone.

The following example illustrates some of the differences between a standard competitive equilibrium and a coarse competitive equilibrium. Let $k=2$ and assume that the utility function is logarithmic. There are four equally likely states and all agents have the same endowment. Table 1 below describes this common endowment $s$, the three coarse consumption plans $\left(c^{1}, c^{2}, c^{3}\right)$ in the support of the equilibrium allocation, the coarse competitive equilibrium price and, finally, the equilibrium price in a standard economy without the coarseness constraint.

Note that in every equilibrium plan, there is a cutoff state; that is, each agent chooses a state $j \in\{1,2,3\}$ such that states $i \leq j$ are associated with low consumption ("bad times") 


\begin{tabular}{cccccc}
\hline \hline endowment & $c^{1}$ & $c^{2}$ & $c^{3}$ & coarse price & standard price \\
\hline 1 & .85 & 1.04 & 1.18 & .40 & .35 \\
$4 / 3$ & 1.7 & 1.04 & 1.18 & .25 & .26 \\
$5 / 3$ & 1.7 & 1.96 & 1.18 & .21 & .21 \\
2 & 1.7 & 1.96 & 2.56 & .13 & .18 \\
\hline
\end{tabular}

Table 1: Equilibrium allocations and prices.

and states $i>j$ are associated with high consumption ("good times"). The last column of Table 1 contains the equilibrium price without the coarseness constraint. In this equilibrium, every agent consumes the aggregate endowment. Note also that the largest difference between the equilibrium price in a standard economy and the coarse competitive equilibrium price is in the extreme states with endowments 1 and 2. In the standard case, the price ratio between those states is 2 , the ratio of the aggregate endowment in those two states. In contrast, the price ratio between those same states is 3 in a coarse competitive equilibrium. As we will show in the next section, this is no accident. In any coarse competitive equilibrium with many states, the prices in states with endowments near the upper or lower bounds differ most from standard equilibrium prices.

Finally, note that in each state, the coarse competitive equilibrium consumption distribution is a mean preserving spread of the corresponding (degenerate) standard competitive equilibrium consumption distribution. More generally, since agents with the same endowment choose different consumption plans in a coarse competitive equilibrium, the equilibrium distribution of consumption in any state of the standard economy second order stochastically dominates the corresponding distribution for the coarse economy whenever all agents have the same endowment.

\section{Extreme Prices}

The four state example of the previous section suggests that the effect of coarseness on equilibrium prices is most pronounced when the realized endowment is exteremly large or extremely small. Theorem 4, our main result, shows that this is true in general. In Theorem 4, we consider an economy with an endowment distribution that approximates a continuous distribution. 
In other words, we consider a sequence of economies with a common utility index $u$, common coarseness constraint $k$, and aggregate endowments that converge to a continuous distribution. Our main result characterizes the limit of the corresponding equilibrium prices near the upper and lower boundary of the endowment range.

Let $E^{n}$ be a pure endowment economy with $n \geq k+1$ states and order states so that $s_{i}<s_{j}$ if $i<j$. A sequence of economies, $\left\{E^{n}\right\}=\left\{\left(u, k, \pi^{n}, s^{n}\right)\right\}$, is convergent if $s^{n}$ converges in distribution to a probability distribution with a continuous and strictly positive density on $[a, b]$. Let $p^{n}$ be the coarse competitive equilibrium price of $E^{n}$. Some of properties of the limit price are more conveniently stated in terms of the pricing kernel; that is, the equilibrium price normalized by the probability of the state. The pricing kernel is

$$
\kappa_{i}^{n}=\frac{p_{i}^{n}}{\pi_{i}^{n}}
$$

Given a convergent sequence of economies, we call the corresponding equilibrium price and pricing kernel sequence, $\left\{\left(p^{n}, \kappa^{n}\right)\right\}$, a coarse competitive equilibrium price sequence.

For any real-valued function $X$ on $\{1, \ldots, n\}$ and $A \subset \mathbb{R}$, let $\operatorname{Pr}(X \in A)$ denote the probability that $X$ takes a value in $A$; that is, $\operatorname{Pr}(X \in A):=\sum_{\left\{i: X_{i} \in A\right\}} \pi_{i}$. To avoid having to say "there exists a subsequence such that" multiple times, below we let $\lim x^{n}$ denote an arbitrary limit point of any bounded sequence $\left\{x^{n}\right\} .{ }^{7}$ Recall that $\rho$ is the coefficient of relative risk aversion.

Theorem 4. For any coarse competitive equilibrium price sequence $\left\{\left(p^{n}, \kappa^{n}\right)\right\}$

(i) $\lim p_{1}^{n}>0$ and, if $\rho \geq 1$, then $\lim \operatorname{Pr}\left(\kappa^{n}>K\right)>0$ for all $K$;

(ii) $\lim \operatorname{Pr}\left(\kappa^{n}<\epsilon\right)>0$ for all $\epsilon>0$ and, if $\rho>1$, then $\lim \operatorname{Pr}\left(p^{n}=0\right)>0$.

For state 1, the state with the lowest endowment, Theorem 4(i) establishes that the limit price is greater than zero even though the limit probability of that state is zero. Thus, consumption in state 1 is extremely expensive. Clearly, this implies that the pricing kernel in state 1 goes to infinity. The second part Theorem 4(i) asserts that if the agent's parameter

\footnotetext{
${ }^{7}$ That is, the claims in Theorem 4 hold for any convergent price subsequence and the sequences in question are bounded.
} 
of relative risk aversion is greater or equal to 1 , the limit distribution of the pricing kernel is unbounded.

Part (ii) of Theorem 4 establishes that there is a positive limit probability that the pricing kernel is arbitrarily close to zero. By Theorem 3, this occurs when the endowment realization is near its upper bound $b$. Moreover, if relative risk aversion is above 1 , a stronger result is true: the limit price is zero with positive probability.

To prove Theorem 4, we first establish the following lemma (Lemma 11): let $U_{*}^{n}$ be the maximal utility of a consumer in $E^{n}$ at the coarse competitive equilibrium prices when restricted to $k$-coarse plans. Let $Y^{n}$ be the maximal utility that the same agent could attain at the same prices if she were restricted to $k-1$-coarse consumptions. Clearly, $Y^{n} \leq U_{*}^{n}$. Lemma 11 shows that $Y^{n}$ is bounded away from $U_{*}^{n}$; hence, $k-1$-coarse plans do uniformly worse than equilibrium plans.

To see the argument for the first part of Theorem 4, assume that the equilibrium price in state 1 converges to zero. In equilibrium, some agents must choose a lower consumption in state 1 than in all other states because aggregate consumption is lower in state 1 than in all other states and because all equilibrium plans are monotone. An alternative plan for these consumers would be to increase consumption in state 1 and make it equal to consumption in state 2 while reducing consumption in the remaining states a bit so as to satisfy the budget constraint. If the price in state 1 converges to zero, then this plan yields essentially the same utility as the original plan, as the compensating reduction in consumption in higher states vanishes. But since the new plan is $k-1$-coarse, we have established a contradiction to Lemma 11. Hence, the price in state 1 must stay bounded away from zero.

A similar application of the Lemma 11 shows that consumption in the highest endowment states must be very cheap so that consumers find it worthwhile to single them out: so cheap that the probability-weighted utility in those states stays bounded away from zero. As a consequence, the pricing kernel must be close to zero. For the final part of Theorem 4 note that utility is bounded above if $\rho>1$ and, therefore, consumers are unwilling to pay attention to very unlikely low-price events no matter how low the price. In that case, part of the aggregate endowment near $b$ is not consumed and prices are zero.

Consider a coarse competitive equilibrium price sequence $\left\{p^{n}, \kappa^{n}\right\}$ and let $P^{n}(r)=\sum_{i: s_{i}^{n} \leq r} p_{i}^{n}$. 
By Helly's selection theorem, the sequence of cumulative distribution functions $\left\{P^{n}\right\}$ has a convergent subsequence. Let $P$ be its limit and let $P^{\prime}$ be the derivate of $P$. We can interpret $\frac{P^{\prime}}{f}$ as the pricing kernel for the limit economy. ${ }^{8}$ If $k=2$ and consumers have a log-utility, we can calculate $\frac{P^{\prime}}{f}$ by solving a differential equation.

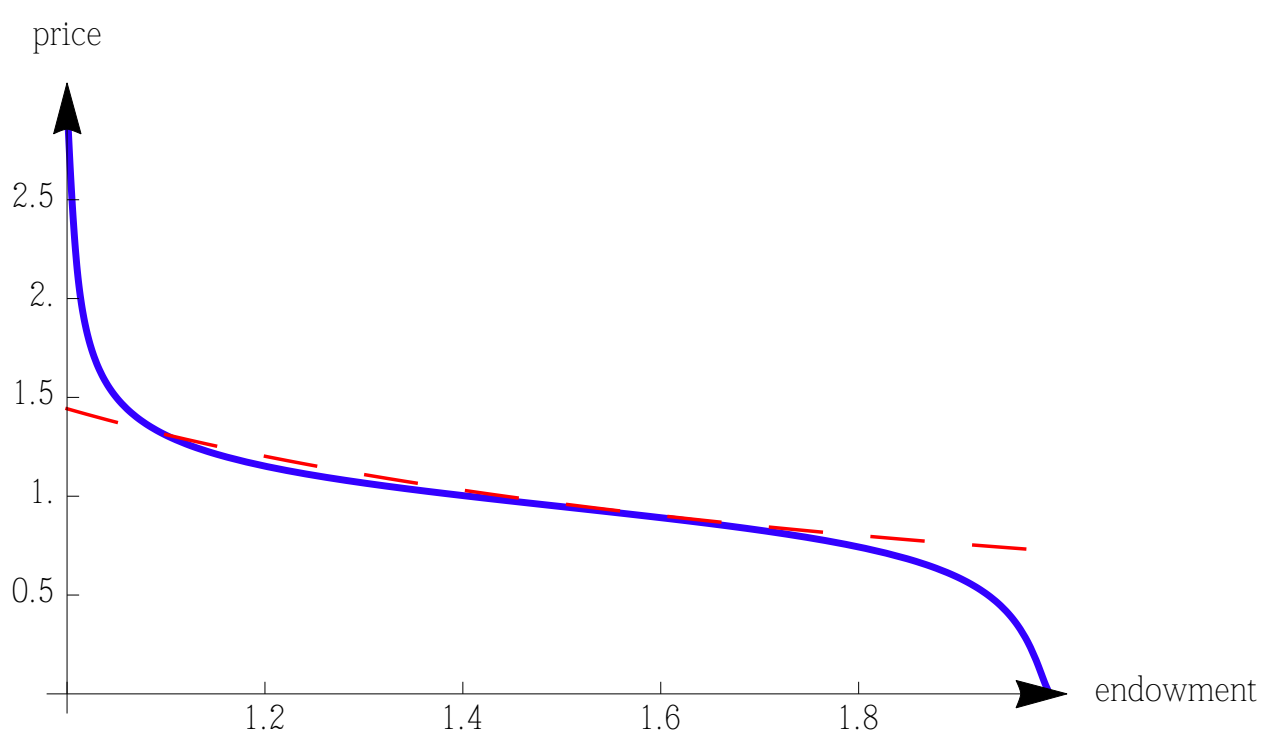

Figure 1: Limit price in coarse competitive equilibrium and in standard economy

Figure 1 depicts the pricing kernel when the limit endowment is uniformly distributed on the interval $[1,2]$ and hence $\frac{P^{\prime}}{f}=P^{\prime}$. The solid line is $P^{\prime}$ for the coarse competitive equilibrium and the dashed line is $P^{\prime}$ in a standard economy without the coarseness constraint. By Theorem 4 , in the coarse competitive equilibrium, $P^{\prime}$ goes to infinity as the endowment converges to the lower bound 1 and to zero as the endowment converges to the upper bound 2 .

\section{$5 \quad$ Robustness and Extensions}

In this section, we examine the robustness of our results. Specifically, we examine how our results would change if agents were differentiated by their risk posture and their coarseness constraint and if, instead of coarse consumption, we assumed coarse net trades. Finally, we discuss how our conclusions depend consumers having constant relative risk aversion. We also briefly discuss an extension of our analysis to infinite horizon economy and asset prices in that

\footnotetext{
${ }^{8}$ Since $P(a)>0$ by Theorem 4 , this interpretation is valid only at $r>a$.
} 
economy.

\subsection{Differentiated Households}

We have assumed that all agents share a common CRRA utility index and a common coarseness constraint. Consider, instead, a model with a finite set of types, each with a type-specific CRRA utility index and a type-specific coarseness constraint. Monotonicity and measurability (Theorem 1) continue to hold for each agent. Moreover, existence of equilibrium can be established using a standard argument for continuum economies. ${ }^{9}$ Because aggregate demand is monotone and measurable, equilibrium prices are monotone; that is,

$$
s_{i}>s_{j} \text { implies } \frac{p_{i}}{\pi_{i}} \leq \frac{p_{j}}{\pi_{j}}
$$

with a strict inequality if $p_{j}>0$. Monotonicity of prices, in turn, implies that in a pure exchange economy all individual consumption plans are monotone and measurable. Of course, the equilibrium allocation is no longer simple. However, simplicity plays no role in the proof of our main theorem, Theorem 4. Thus, a modified version of Theorem 4 would continue to hold: if all types satisfy the corresponding condition on the parameter of risk aversion, then the conclusion of Theorem 4 remains unchanged.

\subsection{Costs Instead of Constraints}

We have assumed that agents cannot adjust their coarseness constraint. Consider, instead, the following extension of our model: each agent can choose $k$ at utility cost $c(k)$. Assume that $c(2)=0$ so that agents have at least 2 elements in their partition. Assume also that $c(k+1)-c(k)>0$ for some $k \geq 2$ and that the marginal cost of relaxing the constraint is increasing; that is, $c(k+1)-c(k)$ is nondecreasing in $k$. The consumer solves the decision problem analyzed in section 2 and chooses $k$ to maximize her overall utility taking into account the utility cost of $k$.

\footnotetext{
${ }^{9}$ The only nonstandard feature of our economy is the nonconvexity of the consumption set. However, our nonconvexity does not jeopardize the existence of a competitive equilibrium. The demand correspondence remains upper hemicontinuous and, therefore, a standard existence argument for continuum economies (Aumann, 1966) applies.
} 
For any fixed value of $k$, individual demand continues to be monotone and measurable. Thus, Theorem 1 would continue to hold under this extension. Moreover, the optimal $k$ would be smaller than $n$, the number of states when $n$ is large and would remain bounded as $n$ goes to infinity. Consumers need not choose the same $k$ in equilibrium; however, this does not affect Theorem 4, as we argued previously. Thus, we conjecture that our main result would extend unchanged to the case in which agents choose $k$ optimally and the utility cost of $k$ satisfies the above conditions.

\subsection{Coarse Net Trades}

As discussed in section 2.2, we require coarse consumption plans but do not restrict agents' ability to sell their endowments (or calculate their budgets). ${ }^{10}$ An alternative model would require coarse net trades; that is, the difference between consumption and endowments would have to be coarse. For example, assuming agents' net trades are 2-coarse amounts to assuming that states are partitioned into "borrowing states" and "lending states" and the agent borrows some fixed amount $x$ if she finds herself in a borrowing state and lends a fixed amount $y$ if she finds herself in a lending state. A model that focuses on coarse net trades may be appropriate for analyzing bounded rationality in portfolio choice. In that case, each agent represents a trader and consumption in a given state is simply the state-contingent money income in that state. In contrast, our model is appropriate for analyzing consumption choices of households that do not respond to every change in price.

In the net-trades model, equilibrium prices are sensitive to the distribution of initial endowments. For example, consider an economy with no aggregate risk but risky individual endowments. Then, the coarse-consumption model has the same equilibrium as the standard model because all agents will consume the same quantity in every state. However, in the net-trades model, consuming the same quantity may be infeasible and hence, the coarse net trade equilibrium may be different than the standard equilibrium. In contrast, if individual endowments are the same (and hence proportional to the aggregate endowment) and there

\footnotetext{
${ }^{10} \mathrm{As}$ an alternative to consumption rigidities, some authors introduce market incompleteness to achieve a similar effect. In Constantinides and Duffie (1996), consumers cannot insure their idiosyncratic income shocks; Krebs (2004) examines a generalization of the Constantinides-Duffie model. Our model delivers an equilibrium in which marginal rates of substitution are not equal across consumers without market incompleteness.
} 
is aggregate risk, then a net-trades model would replicate the standard equilibrium while the coarse-consumption model would not.

Equilibrium prices in the net-trades model depend on the distribution of individual endowments while equilibrium prices of the coarse-consumption model depend only on the aggregate endowment. To generate extreme prices, each model needs its constraint to be binding in equilibrium for many consumers. For the coarse consumption model, this happens whenever there is aggregate risk. To see how the net-trades constraint can be binding, consider the following simple example: every agent has a positive endowment in only one state, the coefficient of relative risk aversion is less than or equal to one, and all agents are $k$-net trade coarse. In this situation, equilibrium prices will be extreme in the sense of Theorem $4 .{ }^{11}$ This example illustrates a more general connection between the coarse-consumption and the coarse-trade model. If endowments are specialized so that for each household the endowment is "far" from the equilibrium consumption plan, then the coarse trade model and the coarse consumption model have similar implications for equilibrium prices. With a single physical good, the simple example above is the most extreme example of specialized endowments. With many physical goods, plausible distributions of endowments will yield a large degree of specialization and, therefore, the coarse net trade model and the coarse consumption model will lead to similar results.

\subsection{Other Utility Functions}

The following example illustrates how equilibrium consumption plans may be nonmonotone when the utility index is not CRRA. Specifically, consider the following utility index:

$$
u(z)= \begin{cases}2 z & \text { if } z \leq 1 \\ 1+z & \text { if } z \in[1,2] \\ 2+z / 2 & \text { if } z>2 .\end{cases}
$$

Table 2 summarizes the unique coarse competitive equilibrium for the aggregate endowment $s=(1,4 / 3,5 / 3,2)$. The table indicates that, in equilibrium, agents choose one of two consump-

\footnotetext{
${ }^{11} \mathrm{~A}$ somewhat more delicate statement will be needed for the $\rho>1$ case to deal with the fact that agents who have all their endowment in the highest aggregate endowment state will end up with zero wealth in equilibrium.
} 
tions plans, $c^{1}$ or $c^{2}$. The fractions in parentheses indicate the fraction of agents choosing the respective plans.

\begin{tabular}{ccccc}
\hline \hline endowment & $c^{1}(2 / 3)$ & $c^{2}(1 / 3)$ & coarse price & standard price \\
\hline 1 & 1 & 1 & .25 & .25 \\
$4 / 3$ & 1 & 2 & .25 & .25 \\
$5 / 3$ & 2 & 1 & .25 & .25 \\
2 & 2 & 2 & .25 & .25 \\
\hline
\end{tabular}

Table 2: Equilibrium allocations and prices

Notice that the second consumption plan $c^{2}$ is not monotone, establishing that with general risk averse utility functions monotonicity may fail. ${ }^{12}$ However, CRRA is not the only class of utility functions that yields monotone consumption plans; Theorem 1 continues to hold if all consumers have the same constant absolute risk aversion.

\subsection{Dynamic Decision Problems}

In this section, we briefly discuss how coarse consumption can be extended to infinite-horizon economies. The formal analysis is in the supplementary online appendix. There, we assume that the aggregate endowment evolves according to a finite state Markov process and consumers maximize discounted expected utility with a CRRA utility index. At the initial stage, each agent partitions the set of possible histories into finitely many categories. The agent chooses the same consumption after any two histories in a given category.

We show in the supplementary appendix that the consumer's optimal attention strategy will partition the range of prices into time-invariant intervals. This characterization allows us to interpret coarse consumption plans as the following two-step optimization problem. In the first step, the consumer identifies $k$ price intervals ${ }^{13}$ with the interpretation that she will not pay attention to price variations within each interval. The consumption choice then solves a

\footnotetext{
${ }^{12}$ The utility function in the example is not strictly concave. However, it is straightforward to show that a strictly concave approximation of the utility function in this example would also lead to nonmonotone equilibrium consumption plans.

${ }^{13}$ The relevant price is the price of consumption after any history appropriately normalized. The appropriate normalization divides the (ex ante) price of consumption by the probability of the particular history and the discount factor. See our online appendix for details.
} 
standard dynamic optimization problem given the chosen price ranges, using the average price for each interval.

Optimal strategies are particularly simple if prices themselves are stationary; that is, if the pricing kernel is the same every time a given state occurs. In that case, each optimal attention strategy uses the same partition of states every period. We show in the online appendix that such stationary equilibria exist and hence, extend coarse competitive equilibria to the infinite horizon economy while retaining the tractability of the two-period model.

\subsection{Safe Haven Premium and Extreme Asset Prices}

In an infinite horizon model with coarse consumers (see the online appendix), we can translate Theorem 4 into a statement about asset prices: in extreme aggregate endowment states (that is, near the upper or near the lower bound) asset prices will also be extreme. Unusually high realizations of the aggregate endowment are associated with extremely high asset prices (expressed in terms of current consumption) while unusually low realizations of the aggregate endowment are associated with extremely low asset prices. This translation of extreme prices for consumption to extreme asset prices enables us to identify the safe haven premium described below.

Consider an asset that is almost risk free; that is, it pays off one unit of consumption with

probability $1-\epsilon$ and pays of nothing with probability $\epsilon$. Suppose that the asset pays zero in states in which consumption is most expensive. Clearly, the risk-free bond that pays off one unit of consumption in all states will trade at a premium over the nearly risk free bond. In a standard competitive equilibrium, this premium converges to zero as $\epsilon$ converges to zero. In contrast, with coarse consumers, this premium stays positive, even in the limit as $\epsilon$ goes to zero. This observation is a straightforward corollary of Theorem 4: in the limit economy with a continuous state space, the price of one unit of consumption in the $\epsilon$-most expensive states stays bounded away from zero for all $\epsilon$. Thus, a risk-free bond differs significantly from a nearly risk-free bond with a very small default risk. We call this difference the safe haven premium. 


\section{Conclusion}

We have analyzed the implications of coarse consumption on equilibrium prices in a standard endowment economy. In particular, we showed that the coarseness constraint leads to extreme and volatile prices when the endowment realization is near its upper or lower bound. Below, we point out the empirical importance of this result.

Many of the empirical puzzles in macroeconomics and finance arise from the difficulty of reconciling the levels of risk aversion implied by equilibrium models with levels that are observed in other contexts or are reasonable a priori (Mehra and Prescott, 1985; French and Poterba, 1991). Our work draws a distinction between the preferences that describe behavior in the absence of any cognitive constraints and observed behavior once those same preferences are filtered through cognitive constraints. We show that the latter behavior may exhibit more risk aversion than the former. In some circumstances, the difference between consumers being very risk averse versus being less risk averse and having a cognitive constraint may be immaterial. However, this difference is important when considering policy interventions that affect cognitive constraints (or costs).

There are a number of other papers that use rigidities in consumption to close the gap between the level of risk aversion needed to rationalize data and plausible levels of risk aversion. Grossman and Laroque (1990) distinguish liquid and illiquid consumption and assume that agents incur transaction costs when they sell an illiquid good. Chetty and Szeidl (2014) focus on the extent to which consumption rigidities reduce stock market participation. Lynch (1996) and Gabaix and Laibson (2002) study a model in which only a fraction of agents can make adjustments at a given time. Unlike those two papers, we do not fix the fraction of agents that can respond to an increase in aggregate output exogenously; rather we require adjustments to be optimal subject to an attention constraint.

The fact that agents choose their partitions optimally enhances the impact of their behavioral limitation on equilibrium prices. This choice renders agents' attention allocation responsive to incentives and hence, endows the market mechanism with a new function: allocating agents' scarce attention. For markets to clear, equilibrium prices must vary sufficiently to attract agents' attention. Since it is particularly costly to pay attention to (unlikely) tail events, the 
price variation near those events has to be large enough to make them salient.

\section{A Appendix: Proofs}

\section{A.1 Proof of Theorem 1}

Let $\sigma=1 / \rho$ and let $S=\left\{S_{1}, \ldots, S_{m}\right\}$ be a partition of $N$ such that $m \leq k$. Let $\mathcal{S}$ be the set of all such partitions. Let $C(S)$ denote the set of all consumptions adapted to the partition $S$; that is, $c \in C(S)$ implies $c_{i}=c_{j}$ whenever $i, j$ are in the same sell of $S$. For any $M \subset N$, let $\pi(M)=\sum_{i \in M} \pi_{i}$ and $p(M)=\sum_{i \in M} p_{i}$. For $c \in C(S)$, we let $c\left(S_{j}\right)=c_{i}$ for $i \in S_{j}$. For $\sigma \geq 1$, $p$ such that $p_{i}>0$ for all $i$, let

$$
W_{\sigma}(S)=\max _{c \in \mathcal{C}(\mathcal{S})} \sum_{j=1}^{k} \pi\left(S_{j}\right) u\left(c\left(S_{j}\right)\right)
$$

subject to $\sum_{i=1}^{m} p\left(S_{j}\right) c\left(S_{j}\right) \leq w$. For $\sigma<1$ and $p$ such that $p_{i} \geq 0$ for all $i$, let

$$
W_{\sigma}(S)=\sup _{c \in \mathcal{C}(\mathcal{S})} \sum_{j=1}^{k} \pi\left(S_{j}\right) u\left(c\left(S_{j}\right)\right)
$$

subject to $\sum_{i=1}^{m} p\left(S_{j}\right) c\left(S_{j}\right) \leq w$. Note that the supremum above is never greater than 0 and is attained if and only if $p\left(S_{j}\right)>0$ for all $j$.

For any $c \in \mathcal{C}_{k}$, let $S^{c}=\left\{S_{1}^{c}, \ldots, S_{m}^{c}\right\}$ denote the partition induced by $c$; that is, the partition such that $i \in S_{t}^{c}$ implies $\left[j \in S_{t}\right.$ if and only if $\left.c_{i}=c_{j}\right]$. Then, the following characterization of optimal consumption plans is straightforward:

$$
U(c)=\max _{c^{\prime} \in B_{k}(p, w)} U\left(c^{\prime}\right) \text { if and only if } U(c)=W_{\sigma}\left(S^{c}\right)=\max _{S \in \mathcal{S}} W_{\sigma}(S)
$$

Define $\psi_{\sigma}: \mathbb{R}_{+} \rightarrow \mathbb{R}$ as follows: $\psi_{1}(t):=t \log (t) ; \psi_{\sigma}(t):=t^{\sigma}$ for $\sigma>1$ and $\psi_{\sigma}(t):=-t^{\sigma}$ for $\sigma<1$. Note that $\psi_{\sigma}$ is strictly convex. For $p$ such that $p_{i}>0$ for all $i$, let $V_{1}(S)=$ $\ln w+\sum_{j} p\left(S_{j}\right) \psi_{\sigma}\left(\pi\left(S_{j}\right) / p\left(S_{j}\right)\right)$ and let $V_{\sigma}(S)=w \sum_{j} p\left(S_{j}\right) \psi_{\sigma}\left(\pi\left(S_{j}\right) / p\left(S_{j}\right)\right)$ for $\sigma>1$. For $p$ such that $p_{i} \geq 0$ and $\sigma<1$, let $V_{\sigma}(S)=w \sum_{j: p\left(S_{j}\right)>0} p\left(S_{j}\right) \psi_{\sigma}\left(\pi\left(S_{j}\right) / p\left(S_{j}\right)\right)$. Notice that $V_{\sigma}$ is defined for all prices if $\sigma<1$ and is defined only for strictly positive prices if $\sigma \geq 0$. Routine calculations for CES utility functions reveal that $V_{\sigma}$ is a monotone transformation of $W_{\sigma}$. 
Lemma 1. Let $a_{i}, b_{i}>0$ for all $i$. (1) Suppose $\frac{a_{3}}{b_{3}} \leq \frac{a_{2}}{b_{2}} \leq \frac{a_{1}}{b_{1}}$ and $\frac{a_{3}}{b_{3}}<\frac{a_{1}}{b_{1}}$. Then, either

$$
\begin{aligned}
& \left(b_{1}+b_{2}\right) \psi_{\sigma}\left(\frac{a_{1}+a_{2}}{b_{1}+b_{2}}\right)+b_{3} \psi_{\sigma}\left(\frac{a_{3}}{b_{3}}\right)>\left(b_{1}+b_{3}\right) \psi_{\sigma}\left(\frac{a_{1}+a_{3}}{b_{1}+b_{3}}\right)+b_{2} \psi_{\sigma}\left(\frac{a_{2}}{b_{2}}\right) \text { or } \\
& b_{1} \psi_{\sigma}\left(\frac{a_{1}}{b_{1}}\right)+\left(b_{2}+b_{3}\right) \psi_{\sigma}\left(\frac{a_{2}+a_{3}}{b_{2}+b_{3}}\right)>\left(b_{1}+b_{3}\right) \psi_{\sigma}\left(\frac{a_{1}+a_{3}}{b_{1}+b_{3}}\right)+b_{2} \psi_{\sigma}\left(\frac{a_{2}}{b_{2}}\right) .
\end{aligned}
$$

(2) Suppose $\sigma<1$ and $\frac{a_{3}}{b_{3}} \leq \frac{a_{2}}{b_{2}}$. Then, either

$$
\begin{aligned}
b_{2} \psi_{\sigma}\left(\frac{a_{1}+a_{2}}{b_{2}}\right)+b_{3} \psi_{\sigma}\left(\frac{a_{3}}{b_{3}}\right) & >b_{3} \psi_{\sigma}\left(\frac{a_{1}+a_{3}}{b_{3}}\right)+b_{2} \psi_{\sigma}\left(\frac{a_{2}}{b_{2}}\right) \text { or } \\
\left(b_{2}+b_{3}\right) \psi_{\sigma}\left(\frac{a_{2}+a_{3}}{b_{2}+b_{3}}\right) & >b_{3} \psi_{\sigma}\left(\frac{a_{1}+a_{3}}{b_{3}}\right)+b_{2} \psi_{\sigma}\left(\frac{a_{2}}{b_{2}}\right) .
\end{aligned}
$$

(3) Suppose $\frac{a_{1}+a_{3}}{b_{1}+b_{3}}<\frac{a_{2}}{b_{2}} \leq \frac{a_{1}}{b_{1}}<\frac{a_{2}+a_{4}}{b_{2}+b_{4}}$. Then, either

$$
\begin{aligned}
& \left(b_{1}+b_{2}+b_{3}\right) \psi_{\sigma}\left(\frac{a_{1}+a_{2}+a_{3}}{b_{1}+b_{2}+b_{3}}\right)+b_{4} \psi_{\sigma}\left(\frac{a_{4}}{b_{4}}\right)>\left(b_{1}+b_{3}\right) \psi_{\sigma}\left(\frac{a_{1}+a_{3}}{b_{1}+b_{3}}\right)+\left(b_{2}+b_{4}\right) \psi_{\sigma}\left(\frac{a_{2}+a_{4}}{b_{2}+b_{4}}\right) \text { or } \\
& b_{3} \psi_{\sigma}\left(\frac{a_{3}}{b_{3}}\right)+\left(b_{1}+b_{2}+b_{4}\right) \psi_{\sigma}\left(\frac{a_{1}+a_{2}+a_{4}}{b_{1}+b_{2}+b_{4}}\right)>\left(b_{1}+b_{3}\right) \psi_{\sigma}\left(\frac{a_{1}+a_{3}}{b_{1}+b_{3}}\right)+\left(b_{2}+b_{4}\right) \psi_{\sigma}\left(\frac{a_{2}+a_{4}}{b_{2}+b_{4}}\right) .
\end{aligned}
$$

(4) Suppose $\sigma<1$ and $\frac{a_{1}+a_{3}}{b_{1}+b_{3}}<\frac{a_{2}}{b_{2}} \leq \frac{a_{1}}{b_{1}}<\frac{a_{2}+a_{4}}{b_{2}}$. Then, either

$$
\begin{aligned}
\left(b_{1}+b_{2}+b_{3}\right) \psi_{\sigma}\left(\frac{a_{1}+a_{2}+a_{3}}{b_{1}+b_{2}+b_{3}}\right) & >\left(b_{1}+b_{3}\right) \psi_{\sigma}\left(\frac{a_{1}+a_{3}}{b_{1}+b_{3}}\right)+b_{2} \psi_{\sigma}\left(\frac{a_{2}+a_{4}}{b_{2}}\right) \text { or } \\
b_{3} \psi_{\sigma}\left(\frac{a_{3}}{b_{3}}\right)+\left(b_{1}+b_{2}\right) \psi_{\sigma}\left(\frac{a_{1}+a_{2}+a_{4}}{b_{1}+b_{2}}\right) & >\left(b_{1}+b_{3}\right) \psi_{\sigma}\left(\frac{a_{1}+a_{3}}{b_{1}+b_{3}}\right)+b_{2} \psi_{\sigma}\left(\frac{a_{2}+a_{4}}{b_{2}}\right) .
\end{aligned}
$$

Proof. To prove part (1), let $D=b_{1}+b_{2}+b_{3}$. Then, let $\hat{p}$ be the lottery that yields $\frac{a_{1}+a_{3}}{b_{1}+b_{3}}$ with probability $\frac{b_{1}+b_{3}}{D}$ and $\frac{a_{2}}{b_{2}}$ with probability $\frac{b_{2}}{D}$. Let $q$ be the lottery that yields $\frac{a_{1}+a_{2}}{b_{1}+b_{2}}$ with probability $\frac{b_{1}+b_{2}}{D}$ and $\frac{a_{3}}{b_{3}}$ with probability $\frac{b_{3}}{D}$. Let $r$ be the lottery that yields $\frac{a_{2}+a_{3}}{b_{2}+b_{3}}$ with probability $\frac{b_{2}+b_{3}}{D}$ and $\frac{a_{1}}{b_{1}}$ with probability $\frac{b_{1}}{D}$. Given the assumptions of the lemma, some straightforward manipulation of inequalities reveals that $q$ is a mean-preserving spread of $\hat{p}$ if $\frac{a_{1}+a_{3}}{b_{1}+b_{3}} \leq \frac{a_{2}}{b_{2}}$ and $r$ is a mean-preserving spread of $\hat{p}$ if $\frac{a_{1}+a_{3}}{b_{1}+b_{3}} \geq \frac{a_{2}}{b_{2}}$. Since $\psi_{\sigma}$ is strictly convex, we conclude that $E_{q}\left[\psi_{\sigma}\right]>E_{\hat{p}}\left[\psi_{\sigma}\right]$ or $E_{r}\left[\psi_{\sigma}\right]>E_{\hat{p}}\left[\psi_{\sigma}\right]$. Each of the preceding two inequalities yields one of the inequalities in the statement of part (1).

To prove part (2), assume $\frac{a_{1}+a_{3}}{b_{3}} \leq \frac{a_{2}}{b_{2}}$. Then, we can replicate the argument in the proof of part (1) to conclude that the first inequality in part (2) holds because $\psi_{\sigma}$ is strictly convex. If $\frac{a_{1}+a_{3}}{b_{3}} \geq \frac{a_{2}}{b_{2}}$, then the second inequality in part (2) holds because $\psi_{\sigma}$ is strictly decreasing.

To prove part $(3)$, let $D=b_{1}+b_{2}+b_{3}+b_{4}$. Then, let $\hat{p}$ be the lottery that yields $\frac{a_{1}+a_{3}}{b_{1}+b_{3}}$ 
with probability $\frac{b_{1}+b_{3}}{D}$ and $\frac{a_{2}+a_{4}}{b_{2}+b_{4}}$ with probability $\frac{b_{2}+b_{4}}{D}$. Let $q$ be the lottery that yields $\frac{a_{1}+a_{2}+a_{3}}{b_{1}+b_{2}+b_{3}}$ with probability $\frac{b_{1}+b_{2}+b_{3}}{D}$ and $\frac{a_{4}}{b_{4}}$ with probability $\frac{b_{4}}{D}$. Let $r$ be the lottery that yields $\frac{a_{3}}{b_{3}}$ with probability $\frac{b_{3}}{D}$ and $\frac{a_{1}+a_{2}+a_{4}}{b_{1}+b_{2}+b_{4}}$ with probability $\frac{b_{1}+b_{2}+b_{4}}{D}$. Finally, let $p=\gamma q+(1-\gamma) r$ where $\gamma=\frac{b_{1}}{b_{1}+b_{2}}$. Under the assumptions of the lemma, $\gamma \in(0,1)$ and the lottery $p$ is a meanpreserving spread of $\hat{p}$. Hence, since $\psi_{\sigma}$ is strictly convex, $E_{p}\left[\psi_{\sigma}\right]>E_{\hat{p}}\left[\psi_{\sigma}\right]$ which means either $E_{q}\left[\psi_{\sigma}\right]>E_{\hat{p}}\left[\psi_{\sigma}\right]$ or $E_{r}\left[\psi_{\sigma}\right]>E_{\hat{p}}\left[\psi_{\sigma}\right]$, proving part (3).

Finally, to prove part (4), let $D=b_{1}+b_{2}+b_{3}$. Then, let $\hat{p}$ be the lottery that yields $\frac{a_{1}+a_{3}}{b_{1}+b_{3}}$ with probability $\frac{b_{1}+b_{3}}{D}$ and $\frac{a_{2}+a_{4}}{b_{2}}$ with probability $\frac{b_{2}}{D}$. Let $q$ be the degenerate lottery that yields $\frac{a_{1}+a_{2}+a_{3}}{b_{1}+b_{2}+b_{3}}$ with probability 1 . Let $r$ be the lottery that yields $\frac{a_{3}}{b_{3}}$ with probability $\frac{b_{3}}{D}$ and $\frac{a_{1}+a_{2}+a_{4}}{b_{1}+b_{2}}$ with probability $\frac{b_{1}+b_{2}}{D}$. Let $p=\gamma q+(1-\gamma) r$, where $\gamma=\frac{b_{1}}{b_{1}+b_{2}}$. Under the assumptions of the lemma, $\gamma \in(0,1)$ and the lottery $\hat{p}$ is strictly second-order stochastically dominates $p$. Since $\psi_{\sigma}$ is strictly convex and strictly decreasing, $E_{p}\left[\psi_{\sigma}\right]>E_{\hat{p}}\left[\psi_{\sigma}\right]$ which means either $E_{q}\left[\psi_{\sigma}\right]>E_{\hat{p}}\left[\psi_{\sigma}\right]$ or $E_{r}\left[\psi_{\sigma}\right]>E_{\hat{p}}\left[\psi_{\sigma}\right]$, proving part (4).

To prove Theorem 1, let $c$ be an optimal consumption plan $c$ and let $S^{c}=\left\{S_{1}^{c}, \ldots S_{m}^{c}\right\}$ and assume w.l.o.g. that $i \in S_{i}^{c}$ for $i=1,2$ and $c_{1}<c_{2}$. Since $c$ is optimal, we must have $W_{\sigma}\left(S^{c}\right)=U(c)$ and therefore $\frac{p\left(S_{1}^{c}\right)}{\pi\left(S_{1}^{c}\right)}>\frac{p\left(S_{2}^{c}\right)}{\pi\left(S_{2}^{c}\right)}$.

First, we will show that (i) $j \in S_{1}$ implies $\frac{p_{j}}{\pi_{j}}>\frac{p\left(S_{2}^{c}\right)}{\pi\left(S_{2}^{c}\right)}$ and (ii) $j \in S_{2}$ implies $\frac{p_{j}}{\pi_{j}}<\frac{p\left(S_{1}^{c}\right)}{\pi\left(S_{1}^{c}\right)}$. If not, if say $p_{1} \cdot \pi(2) \leq p\left(S_{2}^{c}\right) \cdot \pi_{1}$ or $p_{2} \cdot \pi\left(S_{1}^{c}\right) \geq p\left(S_{1}^{c}\right) \cdot \pi_{2}$, then either $\left[S_{1} \backslash\{1\} \neq \emptyset\right.$ and $\left.\frac{p\left(S_{1}^{c}\right)-p_{1}}{\pi\left(S_{1}^{c}\right)-\pi_{1}}>\frac{p\left(S_{2}^{c}\right)}{\pi\left(S_{2}^{c}\right)}\right]$ or $\left[S_{2} \backslash\{2\} \neq \emptyset\right.$ and $\left.\frac{p\left(S_{2}^{c}\right)-p_{2}}{\pi\left(S_{2}^{c}\right)-\pi_{2}}<\frac{p\left(S_{1}^{c}\right)}{\pi\left(S_{1}^{c}\right)}\right]$. In the former case, let $a_{1}=\pi_{1}, b_{1}=p_{1}, a_{2}=\pi\left(S_{2}^{c}\right)$, $b_{2}=p\left(S_{2}^{c}\right), a_{3}=\pi\left(S_{1}^{c}\right)-\pi_{1}$ and $b_{3}=p\left(S_{1}^{c}\right)-p_{1}$ and define the partitions $S=\left(S_{1}, \ldots, S_{m}\right)$, $T=\left(T_{1}, \ldots, T_{m}\right)$ as follows: $S_{1}=S_{1}^{c} \backslash\{1\}, S_{2}=S_{1}^{c} \cup\{1\}, T_{1}=\{1\}, T_{2}=\left(S_{1}^{c} \cup S_{2}^{c}\right) \backslash\{1\}$ and $S_{j}=T_{j}=S_{j}^{c}$ for all $j>2$.

Clearly, $a_{i}>0$ for all $i, b_{3}>0$ and, since $b_{2}=0$ is inconsistent with the existence of an optimal plan, $b_{2}>0$. If $b_{1}=0$, then the assumed existence of an optimal consumption implies $\sigma<1$. Then, an appeal to part (2) of Lemma 1 yields either $V_{\sigma}(S)>V_{\sigma}\left(S^{c}\right)$ or $V_{\sigma}(S)>V_{\sigma}\left(S^{c}\right)$. If $b_{1}>0$, then part (1) of Lemma 1 yields the same contradiction. The proof of (ii) is a mirror image of the preceding argument.

Suppose there is an optimal non-measurable or non-monotone consumption plan $c$; that is, $\frac{p_{1}}{\pi_{1}} \leq \frac{p_{2}}{\pi_{2}}$. By (i) and (ii) above, $S_{1} \backslash\{1\} \neq \emptyset \neq S_{2} \backslash\{2\}, \frac{a_{1}+a_{3}}{b_{1}+b_{3}}<\frac{a_{2}}{b_{2}} \leq \frac{a_{1}}{b_{1}}<\frac{a_{2}+a_{4}}{b_{2}+b_{4}}$ and $a_{i}, b_{j}>0$ for $j \neq 4$ and all $i$, where $a_{i}=\pi_{i}, b_{i}=p_{i}$ for $i=1,2$ and $a_{i}=\pi\left(S_{i-2}^{c}\right)-\pi_{i-2}, b_{i}=p\left(S_{i-2}^{c}\right)-p_{i-2}$ for $i=3,4$. Then, let $S=\left\{S_{1}, \ldots, S_{m}\right\}, T=\left\{T_{1}, \ldots, T_{m}\right\}$, where $S_{1}=S_{1}^{c} \cup\{2\}, S_{2}=S^{c} \backslash\{2\}$, 
$T_{1}=S_{1}^{c} \backslash\{1\}, T_{2}=S^{c} \cup\{1\}$ and $S_{i}=T_{i}=S_{i}^{c}$ for $i>2$. Then, if $b_{4}>0$, part (3) of Lemma 1 establishes that either $W_{\sigma}(S)>W_{\sigma}\left(S^{c}\right)$ or $W_{\sigma}(T)>W_{\sigma}\left(S^{c}\right)$ and if $b_{4}=0$, then the assumed existence of an optimal consumption implies $\sigma<1$ and hence, part (4) of Lemma 1 yields the same conclusion, contradicting the optimality of $c$.

\section{A.2 Proof of Theorem 2}

Let $\Phi \subset \Delta$ be the set of all feasible allocations. If $K(\mu) \subset\left\{c^{1}, \ldots, c^{m}\right\}$, we write $\mu=(\mathbf{a}, \mathbf{c})$ where $\mathbf{a}=\left(\alpha^{1}, \ldots, \alpha^{m}\right), \mathbf{c}=\left(c^{1}, \ldots, c^{m}\right)$ and $\mu\left(c^{l}\right)=\alpha^{l}$ for all $l$. It will be understood that $\mathbf{a}=\left(\alpha^{1}, \ldots, \alpha^{m}\right), \hat{\mathbf{a}}=\left(\hat{\alpha}^{1}, \ldots, \hat{\alpha}^{m}\right)$, and so forth. We write $\delta_{c}$ for the allocation in which all agents consume $c \in \mathcal{C}_{k}$.

Lemma 2. If $\mu$ is feasible and not simple, then there is a simple and feasible $\mu^{\prime}$ such that $W\left(\mu^{\prime}\right)>W(\mu)$.

Proof. Let $\mu=(\mathbf{a}, \mathbf{c})$. If $\mu$ is not simple, there is $c, c^{\prime} \in K(\mu)$ such that $c$ and $c^{\prime}$ conform. Let $c^{*}=\gamma \cdot c+(1-\gamma) c^{\prime}$ where $\gamma=\frac{\mu(c)}{\mu(c)+\mu\left(c^{\prime}\right)}$ and let $\mu^{*}$ be the allocation derived from $\mu$ by replacing $c, c^{\prime}$ with a $\left(\mu(c)+\mu\left(c^{\prime}\right)\right.$ probability of $) c^{*}$. Since, $c$ and $c^{\prime}$ are coarse, so is $c^{*}$ and $\mu^{*}$. Since $u$ is strictly concave, $W\left(\mu^{*}\right)>W(\mu)$. Note that $\left|K\left(\mu^{*}\right)\right|<|K(\mu)|$. If $\mu^{*}$ is simple, we are done. Otherwise, repeat the above argument. Since $K(\mu)$ is finite, this process must terminate with a simple allocation.

Lemma 3. If $\mu$ is feasible, simple but not fair, then there is a feasible, simple and fair $\mu^{\prime}$ such that $W\left(\mu^{\prime}\right)>W(\mu)$ and $\left|K\left(\mu^{\prime}\right)\right| \leq|K(\mu)|$.

Proof. Let $\mu=(\mathbf{a}, \mathbf{c})$, let $x^{l}$ be the certainty equivalent of $c^{l}$ and $\bar{x}^{l}$ be the corresponding constant consumption plan; that is, $u\left(x^{l}\right)=U\left(c^{l}\right)$ and $\bar{x}_{i}^{l}=x^{l}$ for all $i, l$. Also, let $x=\sum_{l=1}^{m} \alpha^{l} x^{l}$ and let $\bar{x}$ be the corresponding constant consumption plan. Let $\hat{\mu}=(\hat{\mathbf{a}}, \hat{\mathbf{c}})$ such that $\hat{\alpha}^{l}=\frac{\alpha^{l} x^{l}}{x}$ and $\hat{c}^{l}=\frac{x c^{l}}{x^{l}}$ for all $l$. Finally, let $\bar{\mu}=(\overline{\mathbf{a}}, \overline{\mathbf{c}})$ such that $\bar{\alpha}^{l}=\alpha^{l}$ and $\bar{c}^{l}=\bar{x}^{l}$ for all $l$. Since $u$ is strictly concave and $\mu$ is not fair, $W\left(\delta_{\bar{x}}\right)>W(\bar{\mu})$. Since $u$ is CRRA,

$$
u^{-1}\left(U\left(\hat{c}^{l}\right)\right)=\frac{x}{x^{l}} u^{-1}\left(U\left(c^{l}\right)\right)=\frac{x}{x^{l}} x^{l}=x ;
$$

hence, $W(\hat{\mu})=W\left(\delta_{\bar{x}}\right)$. By definition, $W(\bar{\mu})=W(\mu)$. Hence, $W(\hat{\mu})>W(\mu)$. By construction $\hat{\mu}$ is fair. It is easy to verify that $\sum_{l} \hat{c}_{i}^{l} \hat{\alpha}^{l}=\sum_{l} c_{i}^{l} \alpha^{l}$ for all $i \in N$ and hence $\hat{\mu}$ is feasible. Clearly, $|K(\hat{\mu})| \leq|K(\mu)|$. 
Lemma 4. A solution to the planner's problem exists and every solution to the planner's problem is simple and fair.

Proof. The allocation $\delta_{c}$ such that $c_{i}=\min _{i} s_{i}$ for all $i$ is feasible. Thus, $\Phi$ is nonempty. Since $\delta_{s}$ second order stochastically dominates any feasible $\mu, W(\mu)<W\left(\delta_{s}\right)$ for every feasible $\mu \in \Phi$. Hence,

$$
W_{k}=\sup _{\mu \in \Phi} W(\mu)
$$

is well-defined. By Lemma 2 and Lemma 3, there exists a sequence of feasible, simple, and fair allocations $\mu^{t}=\left(\mathbf{a}^{t}, \mathbf{c}^{t}\right)$ such that $W\left(\mu^{t}\right) \geq W_{k}-1 / t$ and $\mathbf{a}^{t} \in \mathbb{R}_{+}^{m}$ for all $t$, where $m$ is the cardinality of the set of all partitions of $N$ with $k$ or fewer elements.

By passing to a subsequence, $\mathbf{a}^{t}=\left(\alpha^{1 t}, \ldots, \alpha^{m t}\right)$ converges to some $\mathbf{a} \in \Delta\left(\mathbb{R}_{+}^{m}\right)$. If $c^{l t}$ is unbounded for some $l$, we must have $\alpha^{l}=0$. Let $A \subset N$ be the set of $l$ such that $\alpha^{l} \neq 0$. Then, $A \neq \emptyset$ and $c^{l t}$ is bounded for all $l \in A$. Hence, there exists a subsequence of $\mu^{t}$ along which $c^{l t}$ converges to some $c^{l} \in \mathcal{C}_{k}$ for every $l \in A$.

Let $\mu=(\mathbf{a}, \mathbf{c})$ where $\mathbf{a}=\left(\alpha^{l}\right)_{l \in A}$ and $\mathbf{c}=\left(c^{l}\right)_{l \in A}$. Since $\lim W\left(\mu^{t}\right)=W_{k}$ and each $\mu^{t}$ is fair, $U\left(c^{l t}\right)=W\left(\mu^{t}\right)$. So, by the continuity of $u$, we have $U\left(c^{l}\right)=W_{k}$ for all $l \in A$ and therefore $W(\mu)=W_{k}$. Finally, $\sum_{l \in A} \alpha^{l t} c_{i}^{l t} \leq \sum_{l=1}^{m} \alpha^{l t} c_{i}^{l t} \leq s_{i}$ for all $i, l, t$ and so $\sum_{l \in A} \alpha^{l} c_{i}^{l} \leq \sum_{l=1}^{m} \alpha^{l} c_{i}^{l} \leq$ $s_{i}$ for all $i, l$. Hence $\mu$ is feasible and therefore $\mu$ solves the planner's problem. Then, Lemma 2 and Lemma 3 imply that $\mu$ must be simple and fair.

Lemma 5. An allocation solves the planner's problem if and only if it is a coarse competitive equilibrium allocation.

The proof of Lemma 5 is in the online appendix.

Lemma 6. If $(p, \mu)$ is a coarse competitive equilibrium and $\Sigma_{i}(\mu)<s_{i}$, then $p_{i}=0$.

Proof. If $c \in \mathcal{C}_{k}$, then $\gamma c \in \mathcal{C}_{k}$ for all $\gamma>0$. Since $U$ is strictly increasing, $\sum_{N} p_{i} c_{i}=\sum_{N} p_{i} s_{i}$ for any $c \in B_{k}(p, w)$ that maximizes utility. Therefore, $\sum_{N} p_{i} s_{i}=\sum_{c \in K\left(\mu^{n}\right)} \mu(c) \sum_{N} p_{i} c_{i}=$ $\sum_{N} p_{i} \Sigma_{i}(\mu)$. Since $s_{i} \geq \Sigma_{i}(\mu)$ for all $i$, the lemma follows.

Lemma 7. Any coarse competitive equilibrium price is monotone.

Proof. Suppose $(p, \mu)$ is a coarse competitive equilibrium and $\frac{p_{i}}{\pi_{i}}>\frac{p_{j}}{\pi_{j}}$ for some $s_{i}>s_{j}$. By Theorem $1, c_{i} \leq c_{j}$ for all $c$ with $\mu(c)>0$. Hence, $\Sigma_{i}(\mu) \leq \Sigma_{j}(\mu) \leq s_{j}<s_{i}$ and thus by Lemma $6 p_{i}=0$, a contradiction with $p_{j} \geq 0$. 
Lemma 8. Any coarse competitive equilibrium allocation is monotone.

Proof. Let $\mu$ be a coarse competitive equilibrium allocation and suppose that $s_{i}>s_{j}$. By Lemma $7, \frac{p_{i}}{\pi_{i}} \leq \frac{p_{j}}{\pi_{j}}$ and by Theorem $1, c_{i} \geq c_{j}$ for any $c \in K(\mu)$.

Lemma 9. Any coarse competitive equilibrium allocation is measurable.

Proof. Let $\mu$ be a coarse competitive equilibrium allocation and suppose that $s_{i}=s_{j}$. We need to show that for any $c \in K(\mu)$ we have $c_{i}=c_{j}$. Suppose toward contradiction that this is not the case and w.l.o.g that $c_{i}<c_{j}$. By Theorem 1 this implies that $\frac{p_{i}}{\pi_{i}}>\frac{p_{j}}{\pi_{j}}$ and thus for all other $\hat{c} \in K(\mu)$ we have $\hat{c}_{i} \leq \hat{c}_{j}$. Therefore, $\Sigma_{i}(\mu)<\Sigma_{j}(\mu) \leq s_{j}=s_{i}$ and thus, by Lemma $6, p_{i}=0$, a contradiction with $p_{j} \geq 0$.

\section{A.3 Proof of Theorem 3}

This proof is in the online appendix.

\section{A.4 Proof of Theorem 4}

Let $\left\{E^{n}\right\}=\left\{\left(u, k, \pi^{n}, s^{n}\right)\right\}$ be a convergent sequence of economies. Throughout this proof, we consider an agent with wealth 1 . For the definitions of $S=\left(S_{1}, \ldots, S_{m}\right), C(S), \pi(M), p(M)$, $\psi_{\sigma}, c\left(S_{l}\right), W_{\sigma}$ and $V_{\sigma}$, see section A.1 above.

Let $\left\{\left(p^{n}, \mu^{n}\right)\right\}$ be a coarse competitive equilibrium of $E^{n}$. Call a partition of $S$ of $N$ an optimal partition if it is induced by some optimal consumption $c$; that is, $S=S^{c}$. By Lemmas 8 and 9 each of these cells must have the form $S_{i}=\{j, j+1, \ldots, j+m\}$ for some $j, m$. We let $\left\{S^{n}\right\}=\left\{\left(S_{1}^{n}, \ldots, S_{m}^{n}\right)\right\}$ denote a generic sequence of optimal partitions and let $j^{n}(l)$ denote the maximal element of $S_{l}^{n}$ so that $S_{l}^{n}=\left\{j^{n}(l-1)+1, \ldots, j^{n}(l)\right\}$ (set $\left.j^{n}(0):=0\right)$. Then, with some abuse of notation, we write $\pi^{n}(l)$ instead of $\pi^{n}\left(S_{l}\right), \pi^{n}(l)$ instead of $p^{n}\left(S_{l}\right)$ and $c^{n}(l)$ instead of $c\left(S_{l}\right)$. We let $\left\{(\pi(l), p(l))_{l=1}^{m}\right\}$ denote the limit of $\left\{\left(\pi^{n}(l), p^{n}(l)\right)_{l=1}^{m}\right\}$ if this limit exists. Since we typically pass to convergent subsequences, we often suppress the phrase "if the limit exists."

Lemma 10. (1) $\pi\left(l^{*}\right) p\left(l^{*}\right)>0$ for some $l^{*} \in\{1, \ldots, m\}$. (2) If $p_{j+1}^{n}>0$, then there is an optimal partition such that $j^{n}(l)=j$.

Proof. (1) If $\sum_{l=1}^{m} \pi(l) p(l)=0$, then $V_{\sigma}\left(S^{n}\right)$ converges to its upper bound ( $+\infty$ if $\sigma \geq 1$ and 0 if $\sigma<1$ ) which is inconsistent with feasibility. (2) If there is no optimal partition such that 
$j^{n}(l)=j$, then aggregate demand in state $j$ is equal to aggregate demand in state $j+1$ and, therefore, $p_{j+1}^{n}=0$ by Lemma 6 .

Let $U_{*}^{n}$ be the equilibrium utility of this agent and let $Y^{n}$ be the utility of a $k$-1-coarse agent with wealth 1 . In the online appendix, we prove the following lemma:

Lemma 11. $\liminf _{n}\left[U_{*}^{n}-Y^{n}\right]>0$.

By Lemma 11, for $n$ large enough; that is, for a suitable subsequence of economies, every optimal partition for the economy $E^{n}$ must have exactly $k$ cells, $S_{1}, \ldots, S_{k}$.

Lemma 12. Suppose $\pi(l)=0$. (1) If $\sigma<1$, then $p(l)>0$. (2) If $\sigma \geq 1$, then either $p(l)>0$ or $\lim \frac{p^{n}(l)}{\pi^{n}(l)}=0$.

Proof. By Lemma 10, we can choose $m$ such that $p(m) \pi(m)>0$. Let $\hat{S}^{n}$ be the partition that combines cells $S_{m}^{n}$ and $S_{l}^{n}$. For $\sigma \neq 1$, we have

$$
\begin{aligned}
V_{\sigma}\left(S^{n}\right)-V_{\sigma}\left(\hat{S}^{n}\right) & =p^{n}(m) \psi_{\sigma}\left(\frac{\pi^{n}(m)}{p^{n}(m)}\right)+p^{n}(l) \psi_{\sigma}\left(\frac{\pi^{n}(l)}{p^{n}(l)}\right) \\
& -\left(p^{n}(m)+p^{n}(l)\right) \psi_{\sigma}\left(\frac{\pi^{n}(m)+\pi^{n}(l)}{p^{n}(m)+p^{n}(l)}\right)
\end{aligned}
$$

If the lemma were false, we would have $p(l)=0$ for $\sigma<1$ and, therefore, $\lim p^{n}(l) \psi_{\sigma}\left(\frac{\pi^{n}(l)}{p^{n}(l)}\right)=0$. For $\sigma \geq 1$, we would have $p(l)=0$ and $\lim \sup \frac{p^{n}(l)}{\pi^{n}(l)}>0$. Again, we have $\lim p^{n}(l) \psi_{\sigma}\left(\frac{\pi^{n}(l)}{p^{n}(l)}\right)=$ 0 . Since $p(m) \pi(m)>0$ and $\psi_{\sigma}$ is continuous, $\lim \psi_{\sigma}\left(\frac{\pi^{n}(m)+\pi^{n}(l)}{p^{n}(m)+p^{n}(l)}\right)=\psi_{\sigma}\left(\frac{\pi(m)}{p(m)}\right)$ and hence $\lim V_{\sigma}\left(S^{n}\right)-V_{\sigma}\left(\hat{S}^{n}\right)=0$, contradicting Lemma 11.

Recall that $a$ is the lower bound of the support of the limit endowment distribution.

Lemma 13. Assume $\sigma \leq 1$ and $\lim s_{j^{n}(l)}^{n}=a$ for some $l=1, \ldots, k$. Then, $\lim \frac{p_{j^{n}(l)}^{n}}{\pi_{j^{n}(l)}^{n}}=\infty$.

Proof. By Lemma 10, we can find $m$ such that $p(m) \cdot \pi(m)>0$ and by passing to a subsequence we can assume $p^{n}(m) \cdot \pi^{n}(m)>0$ for all $n$. Since $s^{n}$ converges in distribution to a random variable with a continuous density, the hypothesis of the lemma implies that $\lim \sum_{l^{\prime} \leq l} \pi^{n}\left(l^{\prime}\right)=0$ and therefore, $\lim \pi^{n}\left(l^{\prime}\right)=0$ for all $l^{\prime} \leq l$. Then, for $\sigma<1$, Lemma 12 yields $p(l)>0$. For $\sigma=1$, Lemma 12 yields that either $p(l)>0$ or $\lim \frac{p^{n}(l)}{\pi^{n}(l)}=0$. If the latter is true, then Theorem 3 implies that $p\left(l^{\prime}\right)=0$ for all $l^{\prime}>l$. Hence, we have $\pi\left(l^{\prime}\right)=0$ for all $l^{\prime} \leq l$ and $p\left(l^{\prime}\right)=0$ for all $l^{\prime}>l$, contradicting the fact that $p(m) \cdot \pi(m)>0$. Therefore, $p(l)>0$ in this case as well. 
Assume, contrary to the assertion of the lemma, that $\frac{p_{j^{n}(l)}^{n}}{\pi_{j^{n}(l)}} \leq K<\infty$ along some subsequence. First, consider the $\sigma<1$ case. Let $\hat{S}^{n}$ be the partition derived from $S^{n}$ by moving the state $j^{n}(l)$ from $S_{l}^{n}$ to $S_{m}^{n}$. Then, $V_{\sigma}\left(\hat{S}^{n}\right)-V_{\sigma}\left(S^{n}\right)=x^{n}-y^{n}$, where

$$
\begin{aligned}
& y^{n}:=p^{n}(l)^{1-\sigma} \pi^{n}(l)^{\sigma}-\left(p^{n}(l)-p_{j^{n}(l)}^{n}\right)^{1-\sigma}\left(\pi^{n}(l)-\pi_{j^{n}(l)}^{n}\right)^{\sigma} \\
& z^{n}:=\left(p^{n}(m)+p_{j^{n}(l)}^{n}\right)^{1-\sigma}\left(\pi^{n}(m)+\pi_{j^{n}(l)}^{n}\right)^{\sigma}-p^{n}(m)^{1-\sigma} \pi^{n}(m)^{\sigma}
\end{aligned}
$$

Since $\pi^{n}(l)-\pi_{j^{n}(l)}^{n} \geq 0, p^{n}(l) \geq p^{n}(l)-p_{j^{n}(l)}^{n} \geq 0$ and $\sigma \in(0,1)$, we have

$$
\frac{y^{n}}{\pi_{j^{n}(l)}^{n}} \geq \frac{p^{n}(l)^{1-\sigma}}{\pi_{j^{n}(l)}^{n}}\left(\pi^{n}(l)^{\sigma}-\left(\pi^{n}(l)-\pi_{j^{n}(l)}^{n}\right)^{\sigma}\right) \geq \frac{p^{n}(l)^{1-\sigma}}{\pi_{j^{n}(l)}^{n}}\left(\sigma \frac{\pi_{j^{n}(l)}^{n}}{\pi^{n}(l)^{1-\sigma}}\right)=\sigma\left(\frac{p^{n}(l)}{\pi^{n}(l)}\right)^{1-\sigma}
$$

where the second inequality above follows from the fact that the function $f(x)=\pi^{n}(l)^{\sigma}-$ $\left(\pi^{n}(l)-x\right)^{\sigma}$ is convex and therefore, $f(x) \geq f(0)+x f^{\prime}(0)$. Since $\lim \pi^{n}(l)=0$ and $\lim p^{n}(l)=$ $p(l)>0, \lim \frac{y^{n}}{\pi_{j^{n}(l)}^{n}}=\infty$. Then, since $p_{j^{n}(l)}^{n} \leq K \pi_{j^{n}(l)}^{n}$,

$$
\frac{z^{n}}{\pi_{j^{n}(l)}^{n}} \leq \frac{1}{\pi_{j^{n}(l)}^{n}}\left(\left(p^{n}(m)+K \pi_{j^{n}(l)}^{n}\right)^{1-\sigma}\left(\pi^{n}(m)+\pi_{j^{n}(l)}^{n}\right)^{\sigma}-p^{n}(m)^{1-\sigma} \pi^{n}(m)^{\sigma}\right)
$$

Since $p(m)>0$, the right hand side of this inequality converges to $(1-\sigma) K\left(\frac{\pi(m)}{p(m)}\right)^{\sigma}+\sigma\left(\frac{p(m)}{\pi(m)}\right)^{1-\sigma}$. Thus, $y^{n}-z^{n}>0$ for $n$ large enough and, therefore, $V_{\sigma}\left(S^{n}\right)<V_{\sigma}\left(\hat{S}^{n}\right)$, contradicting the optimality of $S^{n}$.

Next, consider the $\sigma=1$. Then, $V_{\sigma}\left(S^{n}\right)-V_{\sigma}\left(\hat{S}^{n}\right)=\tilde{y}^{n}-\tilde{z}^{n}$, where

$$
\begin{aligned}
& \tilde{y}^{n}:=\pi^{n}(m) \ln \left(\frac{\pi^{n}(m)}{p^{n}(m)}\right)-\left(\pi^{n}(m)+\pi_{j^{n}(l)}^{n}\right) \ln \left(\frac{\pi^{n}(m)+\pi_{j^{n}(l)}^{n}}{p^{n}(m)+p_{j^{n}(l)}^{n}}\right) \\
& \tilde{z}^{n}=\left(\pi^{n}(l)-\pi_{j^{n}(l)}^{n}\right) \ln \left(\frac{\pi^{n}(l)-\pi_{j^{n}(l)}^{n}}{p^{n}(l)-p_{j^{n}(l)}^{n}}\right)-\pi^{n}(l) \ln \left(\frac{\pi^{n}(l)}{p^{n}(l)}\right)
\end{aligned}
$$

if the cell $l$ contains elements other than $j^{n}$; otherwise $\tilde{z}^{n}=-\pi^{n}(l) \ln \left(\frac{\pi^{n}(l)}{p^{n}(l)}\right)$. In the latter case, $\frac{\tilde{z}^{n}}{\pi_{j^{n}(l)}^{n}}=-\ln \left(\frac{\pi^{n}(l)}{p^{n}(l)}\right) \rightarrow \infty$ since $\pi(l)=\lim \pi^{n}(l)=\lim \pi_{j^{n}(l)}^{n}=0$ and $p(l)=\lim p^{n}(l)>0$. In the former case, since the function $f(t)=t \ln \left(t / p^{n}(l)\right)$ is convex, $f(t) \leq f(t-x)+x f^{\prime}(t)$. 
Therefore,

$$
\frac{\tilde{z}^{n}}{\pi_{j^{n}(l)}^{n}} \geq \frac{\left(\pi^{n}(l)-\pi_{j^{n}(l)}^{n}\right) \ln \left(\frac{\pi^{n}(l)-\pi_{j^{n}(l)}^{n}}{p^{n}(l)}\right)-\pi^{n}(l) \ln \left(\frac{\pi^{n}(l)}{p^{n}(l)}\right)}{\pi_{j^{n}(l)}^{n}} \geq \frac{-p^{n}(l)-\ln \left(\frac{\pi^{n}(l)}{p^{n}(l)}\right)}{p^{n}(l)} \rightarrow \infty .
$$

Also, since $p(m) \pi(m)>0$,

$$
\frac{\tilde{y}^{n}}{\pi_{j^{n}(l)}^{n}}=\frac{\pi^{n}(m) \ln \left(\frac{\pi^{n}(m)}{p^{n}(m)}\right)-\left(\pi^{n}(m)+\pi_{j^{n}(l)}^{n}\right) \ln \left(\frac{\pi^{n}(m)+\pi_{j^{n}(l)}^{n}}{p^{n}(m)+K \pi_{j^{n}(l)}^{n}}\right)}{\pi_{j^{n}(l)}^{n}} \rightarrow K \frac{\pi(m)}{p(m)}-\ln \frac{\pi(m)}{p(m)}-1 .
$$

It follows that $\tilde{y}^{n}<\tilde{z}^{n}$ for large $n$ and, therefore, $V_{\sigma}\left(S^{n}\right)<V_{\sigma}\left(\hat{S}^{n}\right)$ for large $n$, contradicting the optimality of $S^{n}$.

Proof of Theorem 4: First, we will show that for any sequence $\iota^{n}$ such that $\lim s_{\iota^{n}}^{n}=a$, there is an optimal partition $S^{n}=\left(S_{1}^{n}, \ldots, S_{k}^{n}\right)$ and an $\hat{l}^{n}$ such that $j^{n}\left(\hat{l}^{n}\right)=\iota^{n}$ for all $n$ large enough. If not, then by Lemma 10, there is a sequence $\iota^{n}$ such that $\lim s_{\iota^{n}+1}^{n}=\lim s_{\iota^{n}}^{n}=a$ and $p_{\iota^{n}+1}^{n}=0$ for all $n$. By passing to a subsequence, we can assume that all $s_{\iota^{n}+1}^{n}$ are in the same cell, $S_{l}^{n}$, of some optimal partition. Then, by Theorem $3, p_{i}^{n}=0$ for all $i \geq \iota^{n}+1$. In the first paragraph of the proof of Lemma 13, we noted that $\lim s_{\iota^{n}}^{n}=a$ and $\iota^{n}=j^{n}(l)$ for all $n$ implies $\pi^{n}\left(l^{\prime}\right)=0$ for all $l^{\prime} \leq l$. Hence, $\pi\left(l^{\prime}\right) p\left(l^{\prime}\right)=0$ for all $l^{\prime}$, contradicting Lemma 10.

Consider an optimal partition $S^{n}=\left(S_{1}^{n}, \ldots, S_{k}^{n}\right)$ such that $j^{n}(\hat{l})=\iota^{n}$ for all $n$. By Lemma 10 , we can choose $l$ such that $\pi(l) p(l)>0$. Hence, $l>\hat{l}$. Then, Theorem 3 yields $0<$ $\pi^{n}(l) \cdot p^{n}(l) \leq \frac{p^{n}(l)}{\pi^{n}(l)} \leq \frac{p^{n}(\hat{l})}{\pi^{n}(\hat{l})}$. Hence, $\frac{p^{n}(\hat{l})}{\pi^{n}(\hat{l})}$ is bounded away from 0 . Set $\iota^{n}=1$ for all $n$. Since $\frac{p^{n}(\hat{l})}{\pi^{n}(\hat{l})}$ is bounded away from 0 , Lemma 12 yields $p_{1}=p(1)>0$, proving first assertion of part (i) of the theorem.

To prove the second assertion of part (i), assume there are $\sigma<1$ and $K$ such that $\lim \operatorname{Pr}\left(\kappa^{n} \leq K\right)=1$. Then, there are $n_{m}, \imath_{m}$ such that $\sum_{i<\imath_{m}} \pi_{i}^{n} \leq 1 / m$ and $\kappa_{\imath_{m}}^{n} \leq 2 K$ for all $n \geq n_{m}$. By passing to a subsequence, we can assume that $\sum_{i<\imath_{n}} \pi_{i}^{n} \leq \epsilon_{n}, \kappa_{\imath_{n}}^{n} \leq 2 K$ for all $n$ and some sequence $\left\{\epsilon_{n}\right\}$ converging to 0 . Hence, $\lim s_{\imath_{n}}=a$ and $\kappa_{\imath_{n}}^{n} \leq 2 K$ for all $n$, contradicting Lemma 13.

To prove the second assertion in part (ii), we first show that for $\sigma<1$ and $n$ large enough, $p_{n}^{n}=0$, where $p_{n}^{n}$ is the equilibrium price in the highest endowment state, $n$, of the economy $E^{n}$. If not, then each price in the sequence $\left\{p_{n}^{n}\right\}$ is strictly positive, which by Lemma 10, implies 
that $\{n\}$ is the $k^{\prime}$ th cell in some optimal partition. Then, since $\lim \pi_{n}=0$, Lemma 12 implies $p(k)>0$. Then, by Theorem $3, \sum_{i<n} p_{i}^{n} \geq \frac{1-\pi_{n}^{n}}{\pi_{n}^{n}} p_{n}^{n}$ for all $n$ and since $\lim p_{n}^{n}=p(k)>0$, we have a contradiction to the fact that the prices sum to 1 .

Let $\iota_{n}$ be the highest endowment state with a nonzero price in the economy $E^{n}$. Clearly, no cell of an optimal partition can be a subset of $\left\{\iota_{n}+1, \ldots, n\right\}$ since the utility maximization problem of an agent with such cell would not have a solution. Hence, by Lemma 10, $S_{k}^{n}=$ $\left\{\iota_{n}, \ldots, n\right\}$ must be a cell in some optimal partition.

Since $\sum p_{i}^{n}=\sum \pi_{i}^{n}=1$ for all $n$, Theorem 3 implies

$$
\sum_{i \geq j} \pi_{i}^{n} \geq \sum_{i \geq j} p_{i}^{n}
$$

for all $n, j$. In particular, the preceding inequality holds for $j=\iota_{n}$. Hence, $\pi(k) \geq p(k)$ and therefore, by Lemma $12, \pi(k)>0$. Since $\lim \pi_{\iota_{n}}^{n}=0, \pi(k)=\lim \operatorname{Pr}\left(p^{n}=0\right)>0$ as desired.

Note that for $\sigma<1$, the second assertion of part (ii) implies the first assertion of part (ii). To prove the first assertion of part (ii) for $\sigma \geq 1$, choose $\imath^{n}<n$ such that $\lim s_{\imath^{n}}=b$, where $b$ is the highest possible aggregate endowment. Since the utility function is unbounded all equilibrium prices must be strictly positive: hence, $p_{\imath^{n}+1}^{n}>0$. Then, by Lemma 10, there is an optimal plan such that $j^{n}(l)=\imath^{n}$ for some $l<k$. Since $\lim \sum_{l^{\prime}>l} \pi^{n}(l)=0$, equation (A1) and Lemma 12 yield $\lim \frac{\sum_{l^{\prime}>p^{n}\left(l^{\prime}\right)}}{\sum_{l^{\prime}>l} \pi^{n}\left(l^{\prime}\right)}=0$.

In fact, we can strengthen the preceding result: let $\imath^{n}$ be a sequence such that $\lim \sum_{i \geq \imath^{n}} \pi_{i}=$ 0 , then $\lim \frac{\sum_{i \geq \imath^{n}} p_{i}^{n}}{\sum_{i \geq \imath} \pi_{i}^{n}}=0$. To see why this is so, note that Lemma 10 ensures that there is some optimal partition $l=\left\{\imath^{n}, \ldots, i\right\}$ and hence, $\lim \frac{\sum_{i \geq n} p_{i}^{n}}{\sum_{i \geq \imath^{n}} \pi_{i}^{n}}=\lim \frac{\sum_{l^{\prime} \geq l^{n}\left(l^{\prime}\right)}}{\sum_{l^{\prime} \geq l^{n}} \pi^{n}\left(l^{\prime}\right)}=0$.

Suppose, there is $\epsilon>0$ such that along some subsequence $\lim \operatorname{Prob}\left(\kappa^{n} \geq \epsilon\right)=1$. Pass to that subsequence. Since the sequence of economies coverages to a limit with a strictly positive density on $[a, b]$, along some subsequence of that subsequence, $\max _{i \in N} \pi_{i}^{n_{m}}<1 / m^{2}$. Again, pass to that subsequence so that $\max _{i \in N} \pi_{i}^{n}<1 / n^{2}$. Then, let $\jmath^{n}=\max \left\{i: p^{n} / \pi^{n} \geq \epsilon\right\}$. By assumption, $\lim _{n} \sum_{i \leq \jmath^{n}} \pi_{i}^{n}=1$. Hence, pass to a subsequence along which $\sum_{i \leq \jmath^{n}} \pi_{i}^{n}<\frac{1}{n}$. Let $\imath^{n}:=\max \left\{i: \sum_{j=i}^{j^{n}} \pi_{j}^{n} \geq \frac{1}{n}\right\}$. Note that $\sum_{i \geq \imath^{n}} \pi_{i}^{n}<\frac{2}{n}+\pi_{\imath^{n}}^{n} \leq \frac{2}{n}+\frac{1}{n^{2}}$. Therefore, $\lim \sum_{i \geq \imath^{n}} \pi_{i}^{n}=0$, and hence, by the argument of the previous paragraph, $\lim \frac{\sum_{i \geq 2^{n}} p_{i}^{n}}{\sum_{i \geq \imath^{n}} \pi_{i}^{n}}=0$. 
Since $\frac{p_{i}^{n}}{\pi_{i}^{n}} \geq \epsilon$ for all $\imath^{n} \leq i \leq \jmath^{n}$, we have

$$
\frac{\sum_{i \geq n^{n}} p_{i}^{n}}{\sum_{i \geq \imath^{n}} \pi_{i}^{n}} \geq \frac{\frac{\epsilon}{n}}{\frac{2}{n}+\frac{1}{n^{2}}} \rightarrow \frac{\epsilon}{2}>0
$$

yielding the desired contradiction.

\section{References}

Abreu, Dilip and Ariel Rubinstein, "The structure of Nash equilibrium in repeated games with finite automata," Econometrica, 1988, 56 (6), 1259-1281.

Ahn, David S and Haluk Ergin, "Framing contingencies," Econometrica, 2010, 78 (2), 655-695.

Aumann, Robert J, "Existence of competitive equilibria in markets with a continuum of traders," Econometrica, 1966, pp. 1-17.

Chetty, Raj and Adam Szeidl, "The Effect of Housing on Portfolio Choice," Working paper, Harvard University, 2014.

Constantinides, George M and Darrell Duffie, "Asset pricing with heterogeneous consumers," Journal of Political economy, 1996, pp. 219-240.

Dekel, Eddie, Barton L Lipman, and Aldo Rustichini, "Representing preferences with a unique subjective state space," Econometrica, 2001, 69 (4), 891-934.

Dow, James, "Search Decisions with Limited Memory," Review of Economic Studies, 1991, $58(1), 1-14$.

Ellis, Andrew, "Foundations for Optimal Inattention," mimeo, 2015.

Epstein, Larry G, Massimo Marinacci, and Kyoungwon Seo, "Coarse contingencies and ambiguity," Theoretical Economics, 2007, 2 (4), 355-394.

French, Kenneth and James Poterba, "Investor Diversification and International Equity Markets," American Economic Review, 1991, 81 (2), 222-226.

Fryer, Roland and Matthew O Jackson, "A categorical model of cognition and biased decision making," The BE Journal of Theoretical Economics, 2008, 8 (1).

Gabaix, Xavier, "A sparsity-based model of bounded rationality," The Quarterly Journal of Economics, 2014, 129 (4), 1661-1710.

- and David Laibson, "The 6D bias and the equity-premium puzzle," NBER macroeconomics annual, 2002, 16, 257-312.

Grossman, Sanford J. and Guy Laroque, "Asset pricing and optimal portfolio choice in the presence of illiquid durable consumption goods," Econometrica, 1990, 58 (1), 25-51. 
Jehiel, Philippe, "Analogy-based expectation equilibrium," Journal of Economic Theory, 2005, 123 (2), 81-104.

- and Dov Samet, "Valuation equilibrium," Theoretical Economics, 2007, 2 (2), 163-185.

Krebs, Tom, "Testable implications of consumption-based asset pricing models with incomplete markets," Journal of Mathematical Economics, 2004, 40 (1), 191-206.

Lynch, Anthony W., "Decision frequency and synchronization across agents: Implications for aggregate consumption and equity return," The Journal of Finance, 1996, 51 (4), 1479-1497.

Mankiw, N.Gregory and Ricardo Reis, "Sticky Information Versus Sticky Prices: A Proposal to Replace the New Keynesian Phillips Curve," Quarterly Journal of Economics, 2002, 117 (4), 1295-1328.

Masatlioglu, Yusufcan, Daisuke Nakajima, and Erkut Y. Ozbay, "Revealed attention," The American Economic Review, 2012, 102 (5), 2183-2205.

Mehra, Rajnish and Edward C. Prescott, "The equity premium: A puzzle," Journal of monetary Economics, 1985, 15 (2), 145-161.

Mengel, Friederike, "Learning across games," Games and Economic Behavior, 2012, 74 (2), 601-619.

Mohlin, Erik, "Optimal categorization," Journal of Economic Theory, 2014, 152, 356-381.

Mullainathan, Sendhil, "Thinking Through Categories," mimeo, 2002.

Neyman, Abraham, "Bounded complexity justifies cooperation in the finitely repeated prisoners' dilemma," Economics Letters, 1985, 19 (3), 227-229.

Piccione, Michele and Ariel Rubinstein, "On the Interpretation of Decision Problems with Imperfect Recall," Games and Economic Behavior, 1997, 20 (1), 3-24.

Rubinstein, Ariel, "Finite automata play the repeated prisoner's dilemma," Journal of Economic Theory, 1986, 39 (1), 83-96.

Sims, Christopher A., "Implications of rational inattention," Journal of Monetary Economics, 2003, 50 (3), 665-690.

Tibshirani, Robert, "Regression shrinkage and selection via the lasso," Journal of the Royal Statistical Society. Series B (Methodological), 1996, pp. 267-288.

Wilson, Andrea, "Bounded memory and biases in information processing," Econometrica, 2014, 82, 2257-2294.

Woodford, Michael, "Inattentive Valuation and Reference-Dependent Choice," mimeo, 2012. 